\title{
A particle-based computational model to analyse remodelling of the red blood cell cytoskeleton during malaria infections
}

\author{
Julia Jäger ${ }^{1,2}$, Pintu Patra ${ }^{1,2}$, Cecilia P. Sanchez ${ }^{3}$, Michael Lanzer ${ }^{3}$, Ulrich S. \\ Schwarz ${ }^{1,2^{*}}$ \\ ${ }^{1}$ Institute for Theoretical Physics, Heidelberg University, Germany \\ ${ }^{2}$ BioQuant-Center for Quantitative Biology, Heidelberg University, Germany \\ ${ }^{3}$ Center of Infectious Diseases, Parasitology, University Hospital Heidelberg, Germany \\ * Michael.Lanzer@med.uni-heidelberg.de and schwarz@thphys.uni-heidelberg.de
}

\begin{abstract}
Red blood cells can withstand the harsh mechanical conditions in the vasculature only because the bending rigidity of their plasma membrane is complemented by the shear elasticity of the underlying spectrin-actin network. During an infection by the malaria parasite Plasmodium falciparum, the parasite mines host actin from the junctional complexes and establishes a system of adhesive knobs, whose main structural component is the knob-associated histidine rich protein (KAHRP) secreted by the parasite. Here we aim at a mechanistic understanding of this dramatic transformation process. We have developed a particle-based computational model for the cytoskeleton of red blood cells and simulated it with Brownian dynamics to predict the mechanical changes resulting from actin mining and KAHRP-clustering. Our simulations include the three-dimensional conformations of the semi-flexible spectrin chains, the capping of the actin protofilaments and several established binding sites for KAHRP. For the healthy red blood cell, we find that incorporation of actin protofilaments leads to two regimes in the shear response. Actin mining decreases the shear modulus, but knob formation increases it. We show that dynamical changes in KAHRP binding affinities can explain the experimentally observed relocalization of KAHRP from ankyrin to actin complexes and demonstrate good qualitative agreement with experiments by measuring pair cross-correlations both in the computer simulations and in super-resolution imaging experiments.
\end{abstract}

\section{Author summary}

Malaria is one of the deadliest infectious diseases and its symptoms are related to the blood stage, when the parasite multiplies within red blood cells. In order to avoid clearance by the spleen, the parasite produces specific factors like the adhesion receptor PfEMP1 and the multifunctional protein KAHRP that lead to the formation of adhesive knobs on the surface of the red blood cells and thus increase residence time in the vasculature. We have developed a computational model for the parasite-induced remodelling of the actin-spectrin network to quantitatively predict the dynamical changes in the mechanical properties of the infected red blood cells and the spatial distribution of the different protein components of the membrane skeleton. Our simulations show that KAHRP can relocate to actin junctions due to dynamical changes in binding affinities, in good qualitative agreement with super-resolution imaging 
experiments. In the future, our simulation framework can be used to gain further mechanistic insight into the way malaria parasites attack the red blood cell cytoskeleton.

\section{Introduction}

Malaria infections cause around 400.000 fatalities per year [1] and most of these are caused by the species Plasmodium falciparum. The main symptoms of this disease are related to the blood stage, when the malaria parasite hides inside a red blood cell (RBC) [2]. There it replicates to produce around 20 offspring that after 48 hours are released into the blood stream with the rupture of the RBC-envelope. During this development, the parasite removes most of the haemoglobin and causes a dramatic remodelling of the RBC-envelope [3]. Remodelling of the cytoskeleton is essential to prevent premature rupture and to establish a system of adhesive knobs. By cytoadhesion to the vascular endothelium, the residency time in the vasculature is increased and the parasite avoids clearance by the spleen. Strikingly, this dramatic transformation process is controlled by the parasite only from a distance, through secretion of proteins that are targeted to the membrane and there incorporation into the existing surface structures of the RBC [4]. However, the exact points of attack and the temporal sequence of these remodeling events are not yet understood.

The main component of the red blood cell cytoskeleton are spectrin tetramers which form a hexagonal network with in average six tetramers converging to the around 35.000 actin protofilaments, each of which is only $36 \mathrm{~nm}$ long [5,6]. Spectrin tetramers consist of two head-to-head attached dimers and have a contour length of approximately $200 \mathrm{~nm}$ without folding of spectrin subunits [7]. With around $10 \mathrm{~nm}$, the persistence length is much shorter than the contour length [8-10]. Therefore the spectrin filaments are strongly coiled and generate a thick layer underneath the membrane [11-14]. Polymer theory predicts an end-to-end distance of $63 \mathrm{~nm}$, which agrees well with the range of 50 to $100 \mathrm{~nm}$ that has been reported experimentally $[5,6]$. However, the exact length distribution of the spectrin tetramers in equilibrium is not known, since all microscopy techniques require sample preparations that tend to stretch the spectrin chains.

The spectrin-actin cytoskeleton provides shear elasticity to the RBC-envelope and is closely connected to the plasma membrane, which provides bending rigidity [15]. Together, these two layers can withstand the harsh conditions that the RBC faces in the vasculature, ranging from the high shear rates in the heart to the strong confinement in the capillaries. In order for this composite to be mechanically stable, many connections are required. These are established not only by the actin junctional complexes at the nodes of the hexagonal spectrin network, but also at the ankyrin complexes at the midpoints of the spectrin tetramers connecting these junctional complexes $[5,6]$ (Figure 1A). Long being considered to be relatively static structures, only recently it has been found that junctional complexes are in fact in a state of continuous turnover, as demonstrated experimentally by actin monomer exchange and the discovery that not all actin filaments are capped [16-18]. Very important in our context, this insight was originally triggered by the finding that malaria parasite mine actin from the junctional complexes, which then is used to build a new transport system of long filaments between the parasite and the RBC-surface $[19,20]$.

RBCs have a typical lifetime of 120 days and senescent but also malaria-infected RBCs (iRBCs) are removed from the circulation mainly at the interendothelial slits of the spleen. It has been found experimentally that the shear modulus of the membrane skeleton of iRBCs increases by one order of magnitude [21-23]. This stiffening but also the growing parasite mass inside make it very difficult to pass the slits. In order to avoid the clearance by the spleen, the malaria parasite has evolved cytoadhesion as a strategy to increase residence time in the vasculature. The parasite-produced 


\section{A Healthy RBC}

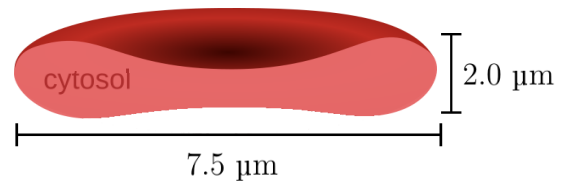

B Late-stage malaria-infected RBC
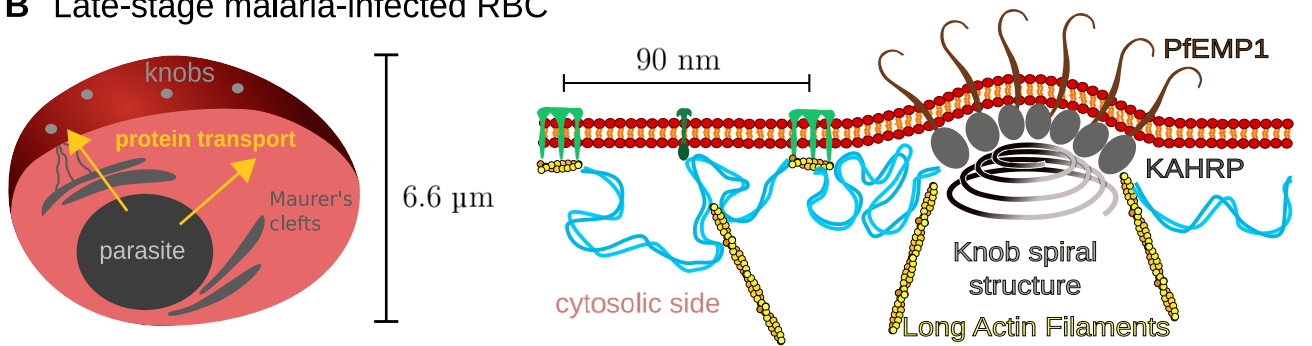

Figure 1. The membrane skeleton in healthy and infected red blood cells. (A) On the left a schematic representation of a cut through a healthy RBC with a biconcave shape is shown. The closeup on the right shows how the actin-spectrin network is coupled to the lipid bilayer through the ankyrin and actin complexes. (B) As shown schematically on the left, in the late stage of the malaria infection the RBC has changed to a spherical shape and adhesive knobs have formed on its surface. The closeup on the right shows the internal structure of the knobs, with a spiral structure at the base, the PfEMP1 adhesion receptors in the membrane at the top and

KAHRP-molecules inbetween. Actin is mined from the junctional complexes and forms long filaments often connected to the Maurer's clefts.

Plasmodium falciparum erythrocyte membrane protein 1 (PfEMP1) is transported along the long actin filaments that result from the actin mining and are clustered in thousands of adhesion clusters called knobs (Figure 1B). These complexes are $100 \mathrm{~nm}$ large protrusions which can be imaged with electron microscopy or atomic force microscopy $[24,25]$. Their main component is the knob-associated histidine rich protein (KAHRP) which is essential for knob formation [26-28]. Moreover it has been shown that a spiral scaffold of unknown composition underlies the knobs [29,30]. Towards the tip of these knobs, on average three PfEMP1 molecules are clustered [31]. As the knobs are formed, also the spectrin network becomes more irregular, with a larger average length of spectrin filaments [32]. Coarse-grained molecular dynamics computer simulations have suggested that the stiffening effect however results mainly from knob formation and less from the changes in the spectrin network [33].

Very recently, we have shown using two-color stimulated emission-depletion (STED) microscopy and image pair cross-correlation analysis that the KAHRP localization dynamcially changes during a malaria infection [34]. During most of the initial ring stage (1-24 hours post invasion), KAHRP co-localizes both with the ankyrin and actin junctional complexes, possibly to create a strong diffusion gradient towards the membrane. At the end of the ring stage and the beginning of the trophozoite stage (24-36 hours post invasion), it re-localizes to the remodelled actin junctional complexes, where most of the knobs seem to form. However, it is unclear which molecular processes are underlying these dynamical changes. Potential mechanisms are changes in KAHRP affinity due to phosphorylation, increases in KAHRP concentration and structural changes in the KAHRP target structures. To explore the spatial aspects of these dynamical changes on the molecular level and how they could explain the known mechanical changes on the cellular level, here we have developed coarse-grained Browninan dynamics simulations that now allow us to quantitatively analyse the 
dramatic remodelling process of RBCs that occurs during the infectious cycle of Plasmodium falciparum. Similar coarse-grained computer simulations have been used before to predict the cellular shear modulus from a microscopic description of the RBC cytoskeleton for healthy and infected RBCs $[33,35,36]$, but not with the focus on the dynamical changes to actin and KAHRP as addressed here.

\section{Materials and methods}

\section{Particle-based model for the cytoskeleton}

The cytoskeletal network is simulated with ReaDDy 2 [37], which is a Brownian Dynamics simulation framework for interacting particles and the additional capabilities to incorporate reactions and filament assembly. Particles diffuse according to an overdamped Langevin equation with predefined potentials depending on nearby particle positions $V\left(\vec{x}_{i}(t), \vec{x}_{j}(t), \ldots\right)$ :

$$
\frac{d \vec{x}_{i}(t)}{d t}=-D_{i} \frac{\nabla V\left(\vec{x}_{i}(t), \vec{x}_{j}(t), \ldots\right)}{k_{B} T}+\vec{\xi}_{i}(t)
$$

where $\vec{x}_{i}(t)$ is the particle position at time $t, D_{i}$ is the particle-specific diffusion constant, $k_{B} T$ is thermal energy and $\vec{\xi}_{i}(t)$ is white noise:

$$
\left\langle\vec{\xi}_{i}(t)\right\rangle=0, \quad\left\langle\vec{\xi}_{i}(t) \vec{\xi}_{i}\left(t^{\prime}\right)\right\rangle=2 D_{i} \delta\left(t-t^{\prime}\right) .
$$

Cytoskeletal filaments and monomers are set up with specific interactions as explained hereafter. The simulation time step was chosen as $\Delta t=0.01 \mathrm{~ns}$ to account for the strong potentials within filaments. A typical simulation run lasts for $0.26 \mathrm{~ms}$.

Filaments are implemented as a connected chain of monomers with harmonic bond potentials between neighbouring beads and angle potentials between three adjacent beads. The pairwise potentials are described in detail in S1 File. Through the angle coefficient $k_{\text {angle }}$ the persistence length $L_{\mathrm{p}}$ of the filament is determined. For the cytoskeletal network of the RBC we need two different types of filaments, spectrin filaments of contour length of $200 \mathrm{~nm}$ and polymerizing actin filaments with an equilibrium length of $36 \mathrm{~nm}$. We use $k_{\text {angle }}=4.28 \mathrm{~kJ} \mathrm{~mol}^{-1}$ for the very flexible spectrin filaments (corresponding to a persistence length of $10 \mathrm{~nm}$ ) and $k_{\text {angle }}=4280 \mathrm{~kJ} \mathrm{~mol}^{-1}$ for the rod-like actin filaments (corresponding to a persistence length of $10 \mu \mathrm{m}$ ). All non-neighbouring particles are subject to a hard-core repulsion to model excluded volume effects. The force constants for the repulsion are given in S1 Table.

A spectrin dimer consists of $21 \alpha$-spectrin repeats, $17 \beta$-spectrin repeats and an actin binding domain at the end as shown schematically in Figure 2A top. Two dimers associate head to head to from a spectrin tetramer. In the simulation the tetramers are implemented as a chain of 39 beads, where the end beads interact with actin and are harmonically confined to a $4 \mathrm{~nm}$ thick plane $10 \mathrm{~nm}$ above the bottom of the simulation box which represents the plane of the lipid bilayer. In the RBC the distance to the bilayer is determined by the spatial dimension of the anchoring proteins. Additionally, the two beads coloured red in Figure 2A bottom are also confined to the plane near the bottom of the simulation box in order to represent the ankyrin bridges.

Actin filaments are implemented with two distinct ends corresponding to the barbed and pointed ends and can react with G-actin to elongate or shrink at predefined rates (compare Figure 2B). The filaments are confined to the $4 \mathrm{~nm}$ thick plane such that they maintain a low angle with respect to the bottom of the simulation box as has been observed in RBCs [13]. Within this plane the filaments are free to diffuse and rotate.

All particles that are confined to the membrane plane are set up with a reduced diffusion constant of $0.53 \mathrm{um}^{2} \mathrm{~s}^{-1}[38]$. The reason is that theses would be connected to 


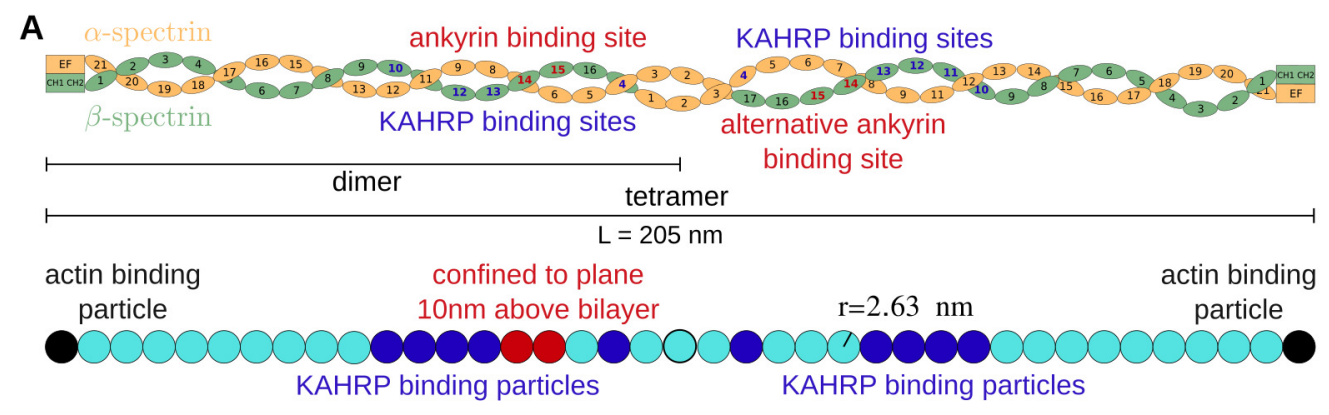

B

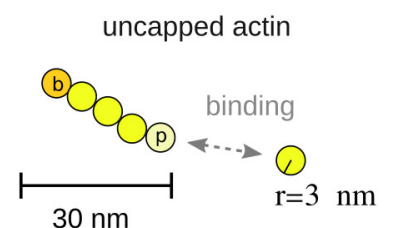

capped actin

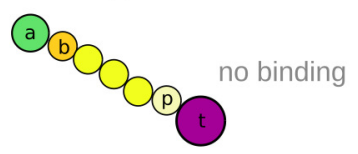

D

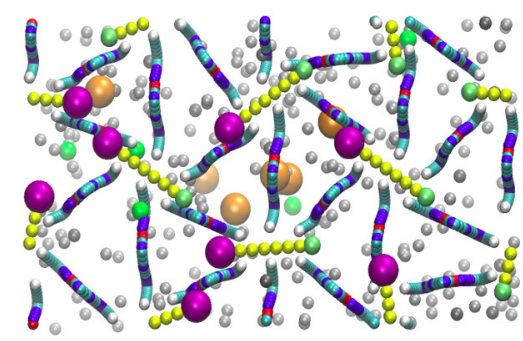

C

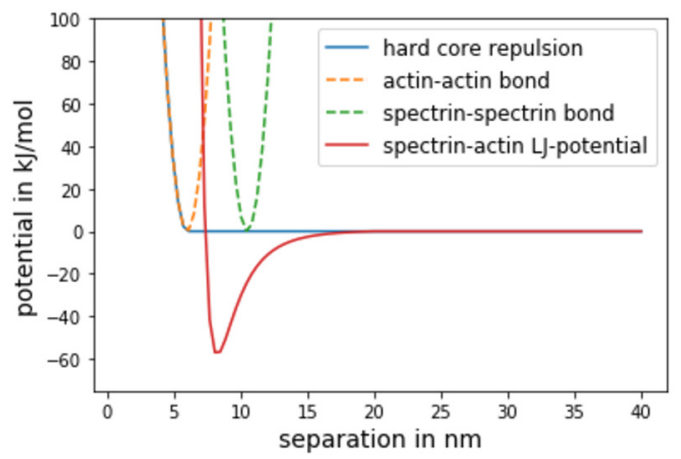

E

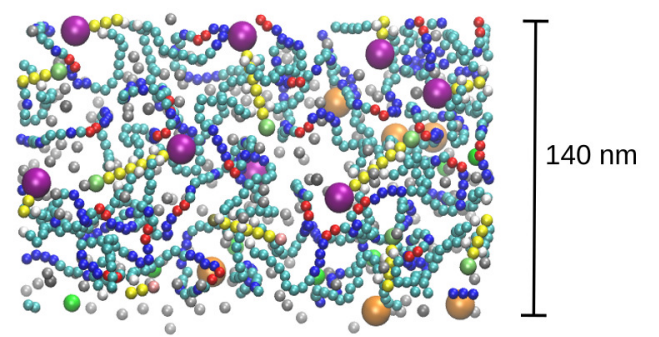

Figure 2. Particle-based model for the RBC cytoskeleton. (A) Schematic of the spectrin tetramer structure at the top and implementation in the simulation at the bottom. Differently coloured beads have different properties that represent the interactions of the spectrin filaments with actin and KAHRP. (B) Implementation of dynamic actin filaments in the simulations. The top filament can polymerize at both ends with different rates at the barbed $(b)$ and pointed $(p)$ ends. For the filament at the bottom the polymerization is blocked, since the capping proteins adducin $(a)$ and tropomodulin $(t)$ are attached. (C) The potentials used in the simulations are plotted against particle separation. (D) The initial configuration of a typical simulation is shown as a projection along the z-direction. The simulation box has periodic boundaries and a size of $140 \times 242.48 \times 100 \mathrm{~nm}^{3}$. An example simulation can be seen in the $\mathrm{S} 1$ Movie. (E) Equilibrated state of the network. 
the lipid bilayer via proteins like anionic exchanger 1 (AE-1, also known as band 3). The reduced value of the diffusion constant is the one measured for band 3 diffusing on small scales within the RBC membrane.

All monomers are assumed to be uniformly reactive and to have an isotropic diffusion constant. The diffusion constant was either taken from previous measurements or calculated from the Stokes-Einstein relation:

$$
D_{i}=\frac{k_{B} T}{6 \pi \eta R_{i}},
$$

with $\eta$ being the dynamic viscosity and $R_{i}$ the hydrodynamic radius of particle $i$. Values are summarised in Table 1. For the cytoskeletal simulations, three kinds of monomers are introduced in order to interact with actin filaments: G-actin, adducin and tropomodulin. G-actin can attach to the barbed or pointed ends and then becomes the new end bead. On the contrary, if one end shrinks, a G-actin monomer is released and the neighbouring particle becomes the end bead. Adducin and tropomodulin block this polymerization process by attaching to the barbed and pointed ends, respectively, and stop any further polymerisation until they detach again (compare Figure 2B). The effect of tropomyosin, which attaches laterally to the actin helix, is not modeled explicitly, but implicitly by enforcing the typical actin protofilament length (see below).

Table 1. Values for collision radii, reaction radii and diffusion constants.

\begin{tabular}{|l|c|c|c|}
\hline particle type & $R_{c}$ in $\mathrm{nm}$ & $R_{i}$ in $\mathrm{nm}$ & $D$ in $\frac{\mathrm{um}^{2}}{\mathrm{~s}}$ \\
\hline G-actin & 3 & 3 & 71.5 \\
adducin & 4.2 & 4.2 & 51.07 \\
tropomodulin & 7.25 & 7.25 & 29.59 \\
Spectrin monomer & 5.26 & 5.26 & 41.1 \\
KAHRP & 2.8 & 2.8 & 76.6 \\
\hline
\end{tabular}

The diffusion constant of G-actin is taken from Lanni et al. [39] and the other values are calculated from the respective molecular masses of the proteins assuming spherical particles and the Stokes-Einstein relation.

In order to model the effect of a malaria infection, we introduce KAHRP particles which can bind to cytoskeletal components and other KAHRP particles via Lennard-Jones potentials (see S1 File for more details), such that they can cluster in the cytoskeletal network and also form large aggregates as found in experiments [27]. The exact position of the KAHRP interaction sites is based on the experimentally observed association properties of KAHRP, which have been found to be the following:

- the spectrin-actin-protein 4.1 junction $[27,40]$

- $\alpha$ spectrin repeat 4 [41] and $\beta$ spectrin repeats 10-14 [28,42,43] (with $\mathrm{K}_{\mathrm{D}}=50 \pm 15 \mu \mathrm{M}[28]$ ) which lie directly next to the spectrin-ankyrin interaction site

- band 3 binding domain of ankyrin [43] (with $\mathrm{K}_{\mathrm{D}}=1.3-1.8 \mu \mathrm{M}[44]$ ).

The binding sites along the spectrin molecule are highlighted in dark blue in Figure 2A and in the simulation snapshots presented later. Since the dissociation constant with spectrin repeats is much larger than the one with ankyrin, we neglect the binding to the blue beads and consider binding to ankyrin as binding to the red beads in Figure 2A. Note that we do not model ankyrin explicitly but KAHRP binds directly to the ankyrin binding site on the spectrin molecule. We also fix the KAHRP 
self-association interaction at a large enough value, such that small clusters can form in the cytosol. Therefore, we are left with two main parameters, the interaction energy between KAHRP and actin $\left(\epsilon_{K A H R P-a c t i n}\right)$ and the interaction energy between KAHRP and ankyrin $\left(\epsilon_{K A H R P-a n k y r i n}\right)$. These binding energies are the depths of the respective Lennard-Jones potentials (Figure $2 \mathrm{C}$ ). When interpreted as the binding free energy $\Delta G$, one can relate them to the dissociation constant through thermodynamic considerations [45]:

$$
\Delta G=k_{B} T \ln \frac{K_{d}}{C},
$$

with $\mathrm{C}$ being a reference concentration and $K_{d}$ the dissociation constant.

\section{Actin dynamics}

In contrast to KAHRP binding via a Lennard-Jones potential, we model the actin binding dynamics as explicit reactions, such that filaments can be formed as topologies in ReaDDy 2. When incorporating reactions into a Brownian Dynamics simulation, a distinction has to be made between macroscopic and microscopic reaction rates, where the diffusive process of finding a reaction partner is included in the macroscopic association rate $k^{+}$. The macroscopic and microscopic rates are related by [37]

$$
k^{+}=4 \pi\left(D_{1}+D_{2}\right)\left[R_{12}-\sqrt{\frac{D_{1}+D_{2}}{k_{\text {micro }}^{+}}} \tanh \left(R_{12} \sqrt{\frac{k_{\text {micro }}^{+}}{D_{1}+D_{2}}}\right)\right],
$$

where $D_{1 / 2}$ are the diffusion constants of the two reaction partners and $R_{12}$ is their separation.

Since the diffusion time scale is much faster than the reaction time scale, in order to obtain reasonable simulation times we use a factor $b$ that speeds up the elongation rate $\omega$, as introduced in [46], and indicate the scaled rates by a hat symbol:

$$
\begin{aligned}
& \hat{\omega}=b \omega=b\left(k^{+} C+k^{-}\right)=\hat{k^{+}} \hat{C}+\hat{k^{-}}, \\
& \hat{k^{+}}=k^{+} b_{k}, \hat{C}=\frac{b}{b_{k}} C \text { and } \hat{k^{-}}=b k^{-},
\end{aligned}
$$

where $\mathrm{C}$ is the concentration which determines the association and $k^{-}$is the dissociation rate. In order to observe actin polymerisation within accessible simulation times, the scaling values are chosen to be $b=100000, b_{k}=200$ and $\hat{C}=500 C$ unless otherwise stated. The parameters are chosen according to experimentally determined values if known. These values and the scaled value after accelerating the polymerization are given in the S2 Table. The length regulation of the actin filaments is implemented through modelling capping proteins and introducing specific length dependent rules for the monomer dissociation. To prevent the filaments and hence the network from disassembling completely, the dissociation is stopped at two monomers introducing a boundary for the modelled process.

In order to mimic the attachment of tropomyosin which has the length of six monomers, the dissociation rates are chosen differently for filaments that are longer than six monomers or shorter. Specifically, the following rules are applied:

- G-actin dissociation at barbed and pointed ends:

for $n>6$ normal rates apply

for $n \leq 6$ the rates are halved

- adducin dissociation rate always the same 
- tropomodulin dissociation:

for $n>6$ the rate in absence of tropomyosin is used

for $n \leq 6$ the rate with tropomyosin present is used

- association rates are not length dependent but depend on concentration

- tropomodulin and adducin block polymerization while attached.

We first set up a hexagonal array of spectrin tetramers (Figure 2D). In the z-direction the filaments initially form a sine curve with the red ankyrin attachment sites placed in the plane at $z=10 \mathrm{~nm}$. This is necessary since the tetramer contour length of $205.14 \mathrm{~nm}$ is much longer than the typical actin-actin separation of 60 to $80 \mathrm{~nm}$. The actin filaments at the junctional points are randomly oriented within the plane at $z=10 \mathrm{~nm}$ and the spectrin filament ends are placed at one of the six positions along both sides of the actin filament. Monomer positions are chosen randomly in the 3D space above the cytoskeletal layer. The network is then relaxed to its equilibrium state (Figure 2E). Simulation images were produced with VMD [47].

\section{Pair cross-correlation analysis}

We use a two-dimensional pair cross-correlation analysis to quantify the co-localization of KAHRP with the other components of the network as recently used for

super-resolution microscopy data $[34,48,49]$. First, we generate a distribution profile for each protein by adding a two-dimensional Gaussian distribution on their 2D projected coordinates obtained from the simulations. The standard deviation of the Gaussian distribution is set to $16 \mathrm{~nm}$ (corresponding to $38 \mathrm{~nm}$ full-width at half-maximum of the used STED-microscope). Using the distribution profile, we create an image for each protein with $1 \mathrm{~nm}$ pixel size. For two images, $I_{R}(x, y)$ (red channel for KAHRP) and $I_{G}(x, y)$ (green channel for another protein), the two-dimensional pair cross-correlation (PCC) between them is given by

$C(r, r+\Delta r)=\frac{A_{\text {image }}}{\pi \Delta r(2 r+\Delta r)} \sum_{\rho=r}^{\rho=r+\Delta r} \frac{\sum_{i, j} \sum_{m, n} I_{R}\left(x_{i}, y_{j}\right) I_{G}\left(x_{m}, y_{n}\right) \delta\left(\left|\mathbf{r}_{\mathbf{i j}}-\mathbf{r}_{\mathbf{m n}}\right|-\rho\right)}{\sum_{i, j} \sum_{m, n} I_{R}\left(x_{i}, y_{j}\right) I_{G}\left(x_{m}, y_{n}\right)}$

where $A_{\text {image }}$ is the area of the image and $\Delta r=6 \mathrm{~nm}$ is the width of the radial bins.

\section{Results}

\section{Simulation of spectrin and actin filaments}

We first examined the polymer properties of a single spectrin filament and vary its length and subunit properties. Excluded volume effects and an angular potential between three neighboring beads for bending stiffness are included as specified in the S1 File. We first simulated single spectrin filaments with an angle constant

$k_{\text {angle }}=4.28 \mathrm{~kJ} \mathrm{~mol}^{-1}$, calculated the time average of the end-to-end distance and compared the result to known polymer models in Figure 3B. Each point is an average over 20 million time steps of size $0.1 \mathrm{~ns}$. The end-to-end distance is defined as the distance between the two end beads coloured black in Figure 3A. In Figure 3B three different types of filaments are compared, the full spectrin model in wine colour, a polymer without excluded volume but with an angle potential in red, and a polymer without any interactions except the harmonic potential connecting neighbours in orange. We see that the spatial extend of the polymer increases with the number of segments and is well-described by the polymer model appropriate for each case. 


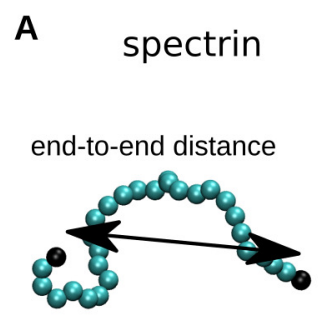

C

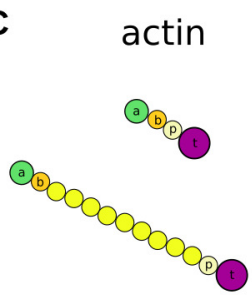

B

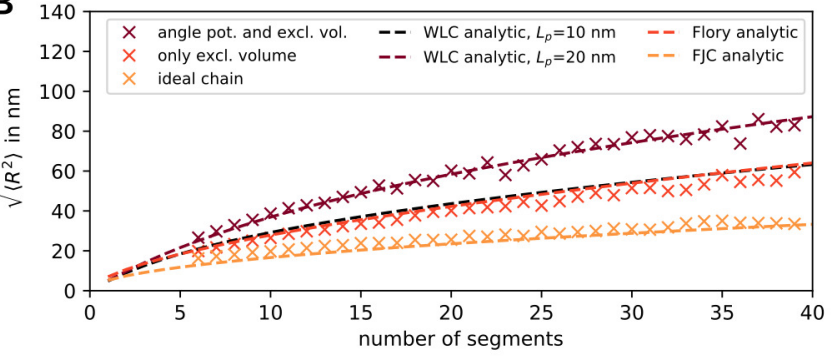

D

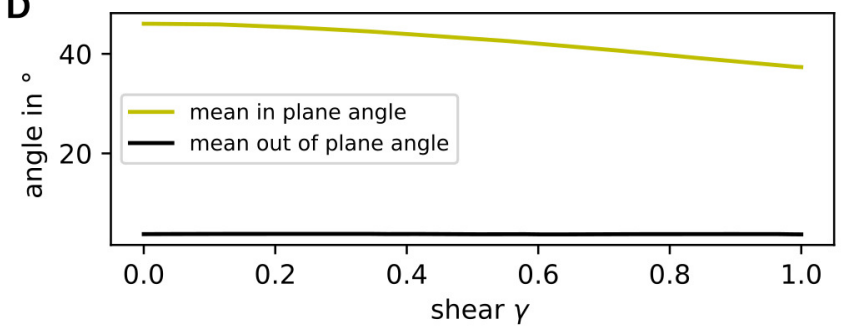

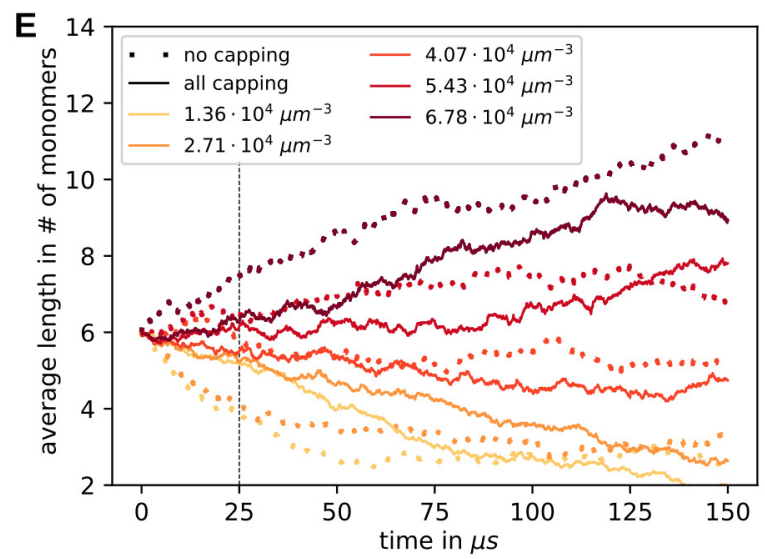

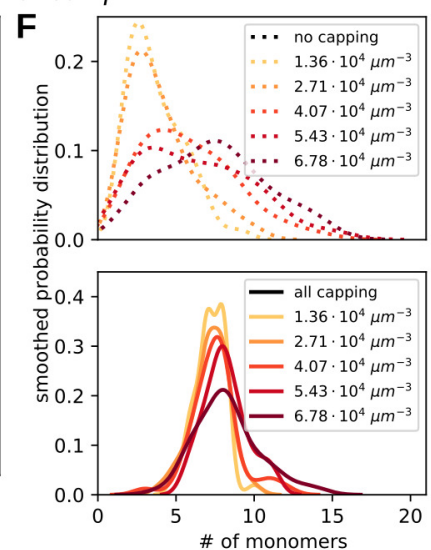

Figure 3. Spectrin and actin filaments. (A) Spectrin properties are tested by examining the end-to-end distance exemplified by the double arrow. (B) The root-mean-square value of the end-to-end distance is calculated for varying filament length. Simulations with an angle potential and excluded volume (wine) are compared to ones without angular confinement in red and simulations of ideal chains (i.e. no angle or excluded volume interactions) in orange. Additionally, we plot the analytic expression for the worm-like chain (wine), the Flory theory (red) and the freely-joined chain (orange). (C) Capped actin filaments of different lengths. (D) The orientation of actin filaments within a network is quantified by the in-plane angle (orientation within the membrane) and the out-of-plane angle (angle in z-direction). These are plotted as a function of shear of the network. (E) The temporal evolution of actin filament average length is plotted for different initial values of G-actin concentration. The solid line shows the data from simulations containing capping proteins and the dotted lines show data from simulations without capping proteins. Each line corresponds to one simulated network with 46 actin filaments. The dashed vertical line indicates the time point for which the distributions are shown in E. (F) The smoothed probability distributions of the filament lengths at time $25 \mu \mathrm{s}$ are shown for all concentrations. At the top the data is shown for simulations without capping proteins and at the bottom with capping proteins. 
The full model corresponds to a worm-like chain with a fitted persistence length of $20 \mathrm{~nm}$, which is a factor of two larger than the theoretical prediction of the worm-like chain model because this model does not incorporate excluded volume effects. The red data points from simulations solely including excluded volume effects can be described by the Flory model and the filaments without interactions mimic the analytically calculated behaviour of a freely-jointed chain model. In the last two cases, the swelling of the polymer is of purely entropic origin, whereas the full model also includes energetic considerations. This analysis also shows that a full spectrin filament containing 39 beads will have an end-to-end distance of $87 \mathrm{~nm}$ in equilibrium and it will be in a highly coiled state if not extended by force.

Going from the single spectrin filament to the full network, we next examine the network thickness, which is approximated by the distance in z-direction (vertical direction in the projection in S1 FigD) between the lowest and highest cytoskeletal bead. It can be seen that the spectrin tetramers are extended away from the bottom of the simulation box which can be thought of as the confining lipid bilayer. The simulated healthy network has an average thickness of $54.21 \mathrm{~nm}$ after equilibration. In S1 FigE the distribution of the different particles along the z-direction can be seen. The actin filaments are confined to a thin section near the bilayer, whereas the spectrin filaments are shown to be confined to a $58 \mathrm{~nm}$ thick plane which is less dense at its edges. The obtained values matches the experimentally determined thickness of approximately $50 \mathrm{~nm}$ of the dense cytoskeletal layer [14]. The experiments showed an additional $40 \mathrm{~nm}$ softer regime which is likely caused by defects in the network that are not present in the simulation.

In the next step we examine the modelled actin filaments, which appear as straight rods because of the much larger persistence length (see Figure 3C). Therefore, we can examine their orientation within the cytoskeletal network as we apply a shear to the network with a shear rate of $3 \cdot 10^{5} \mathrm{~s}^{-1}$. Initially, the filaments are set up parallel to the bilayer plane but randomly oriented within this plane. We can quantify this by two angles, the out of plane angle $\phi$ and the in plane angle $\theta$ (compare S1 FigA). Since we do not take into account the polarity for this analysis, the angles can vary between $0^{\circ}$ and $90^{\circ}$. We now quantify how the angles change when going from the equilibrated reference state to the final state after shearing in x-direction of full-cytoskeleton simulations with $a=88 \mathrm{~nm}$ (see Figure 3D). It can be clearly seen how the average filament angle of all filaments in ten simulations is reduced as a function of shear. This means that the filament angles are not randomly distributed after the shearing, but they align in the shear direction. At the same time the angle in z-direction stays close to its small value. This angle is very close to zero because of the confining potential mimicking the attachment to the lipid bilayer. Histograms of this data can be seen in S1 FigB and C.

Depending on the G-actin concentration and the capping rates, the filaments can grow, shrink or stay at a constant length. The actin dynamics was sped up by a factor $b$ as explained in the Materials and methods section to reach reasonable modelling time scales. The change of actin filament lengths was examined by two types of simulations: one set was conducted without any capping proteins present ("no capping" in Figure 3E and $\mathrm{F}$ ) and the other set contained capping proteins and special rules to mimic tropomyosin attachment in the simulations ("all capping" in Figure $3 \mathrm{E}$ and $\mathrm{F}$ ). The average filament length and the filaments' smoothed probability distributions were examined for different concentrations of free actin monomers $\left(1.36 \cdot 10^{4} \mathrm{\mu m}^{-3}\right.$ to $6.78 \cdot 10^{4} \mathrm{\mu m}^{-3}$ ) in Figure $3 \mathrm{E}$ and $\mathrm{F}$, respectively. We use a hexagonal set-up for the actin filaments as shown in Figure 2D and fix the lattice constant at $a=88 \mathrm{~nm}$.

In Figure $3 \mathrm{E}$ we see how the average filament length grows or shrinks depending on the initial concentration of G-actin. Capping does not completely suppress actin dynamics, but makes it much slower, such that the length stays close to the initial 
length of six monomers for a longer time. To see this in more detail, the filament length distribution was plotted for both cases at time point $25 \mu \mathrm{s}$ in Figure 3F. The

distributions for simulations without capping proteins are clearly much broader than the ones with capping proteins. In both cases the distributions for higher concentrations are spread out further because macroscopic association rates depend on concentration.

\section{Shear modulus}

Next we set up a network of spectrin filaments which are connected via actin filaments at the junctional points and include various diffusing monomers as explained in the Materials and methods section. First, we examine the properties of the simulated cytoskeletal network without any additional malaria-associated alterations in order to validate our novel modelling approach. For examining the mechanical properties of the system, we set up a series of simulations (snapshots shown in Figure 4A-C) mimicking a shear experiment with a shear rate of $3 \cdot 10^{5} \mathrm{~s}^{-1}$ and extract the stress response as seen in Figure 4D-H (an example of the shear simulation can be seen in the S2 Movie). The curves show the mean of ten independent stress responses each.

In order to compare our full model including actin filaments, spectrin persistence and anchoring by band 3 to previous approaches $[33,35,50]$, we set up a reduced system with actin filaments replaced by single particles (compare $4 \mathrm{~A}$ in contrast to the full model in $4 \mathrm{~B}$ ), spectrin filaments with just excluded volume and free diffusion of the actin particles in the bilayer plane. The sheared networks have a size of three hexagonal units times four hexagonal units and vary in size depending on the lattice constants. We set up regular networks with actin particles positioned according to a uniform hexagonal network with lattice constant $a=60-100 \mathrm{~nm}$ and compare their stress responses in Figure 4D. The network with $a=100 \mathrm{~nm}$ displays a shear modulus of $10.92 \mathrm{\mu N} \mathrm{m}^{-1}$ at low shear and $21.22 \mathrm{\mu N} \mathrm{m}^{-1}$ at high shear as extracted from Figure 4D. We also see that for smaller lattice constant $a$ the strain-hardening behaviour of the network is reduced drastically in our model. Previous simulations which modeled actin filaments as one spherical bead also showed a monotonic increase in stress and yielded a modulus of $10-12 \mathrm{\mu N} \mathrm{m}^{-1}$ for small shear at similar shear rates $[33,35,50]$. Moreover this earlier work also predicted shear stiffening for shear values larger than 0.5 . In Figure $4 \mathrm{G}$ we show how the stress changes when we introduce the spectrin persistence and the reduced diffusion of actins. Looking at the curve for $a=100 \mathrm{~nm}$, we see that for small shear the response is similar, but for larger shear rate the shear modulus increases to $23.25 \mathrm{\mu N} \mathrm{m}^{-1}$. Considering the simulations with smaller lattice constant, the shear modulus is elevated at low shear.

In Figure $4 \mathrm{E}$ and $\mathrm{H}$, the stress-shear response is shown for simulations containing explicit actin filaments rather than only actin beads. In $\mathrm{E}$ the spectrin filaments do not have persistence and actin filaments can diffuse freely in the bilayer plane, similar to conditions in $\mathrm{D}$. In $\mathrm{H}$ the response of the full model with persistence and anchoring is shown. The main new feature that appears in these stress responses is a stress jump at very low shear, similar to the elastic response of dense polymer networks [51]. After this initial response the shear modulus is given by $20.8 \mathrm{\mu N} \mathrm{m}^{-1}$ and for high shear the value increases to $49.81 \mathrm{\mu N} \mathrm{m}^{-1}$. Hence, the shear modulus is approximately doubled with respect to the previously examined networks which model the actin filaments as one particle. The stress jump is caused by the newly introduced actin filaments, where the reduced diffusion of these makes the jump even higher as seen in Figure $4 \mathrm{H}$ compared to E. The shearing in these simulations was done at shear rate $3 \cdot 10^{5} \mathrm{~s}^{-1}$, matching the value of previous simulations. However, when we reduce this shear rate, the jump gets smaller and eventually vanishes (see S2 Fig). However, for smaller shear rates the stress magnitude is also reduced in general, as expected.

Recent findings imply that the spectrin network deviates considerably from the ideal 
bioRxiv preprint doi: https://doi.org/10.1101/2021.10.04.462981; this version posted October 4, 2021. The copyright holder for this preprint (which was not certified by peer review) is the author/funder, who has granted bioRxiv a license to display the preprint in perpetuity. It is made available under aCC-BY 4.0 International license.
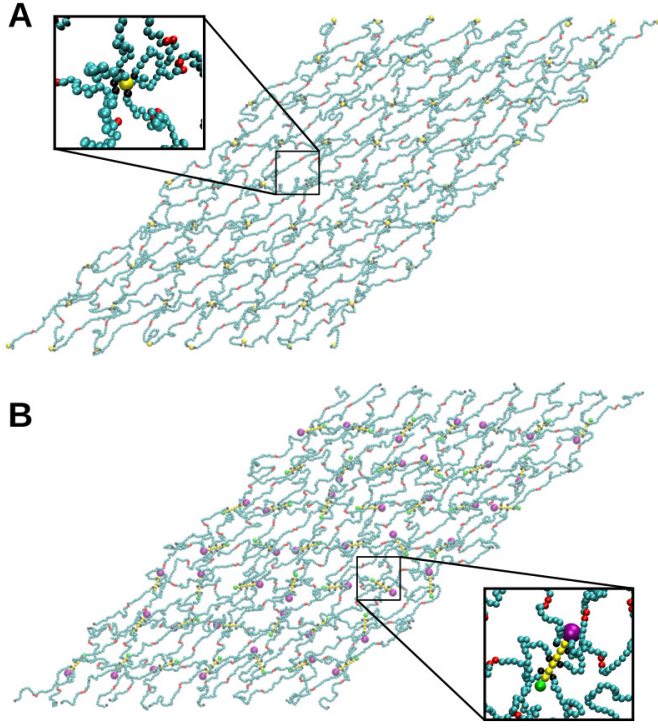

C

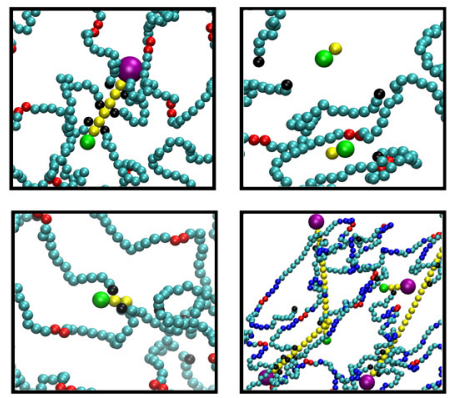

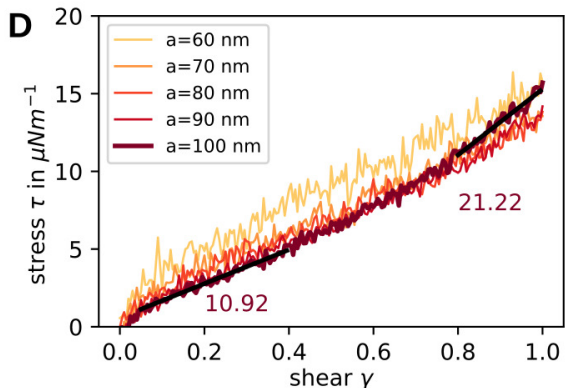
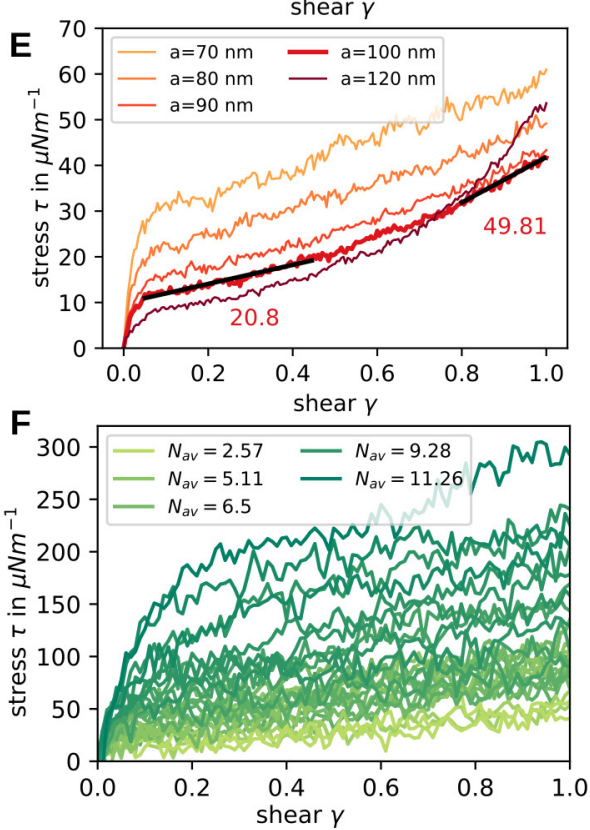
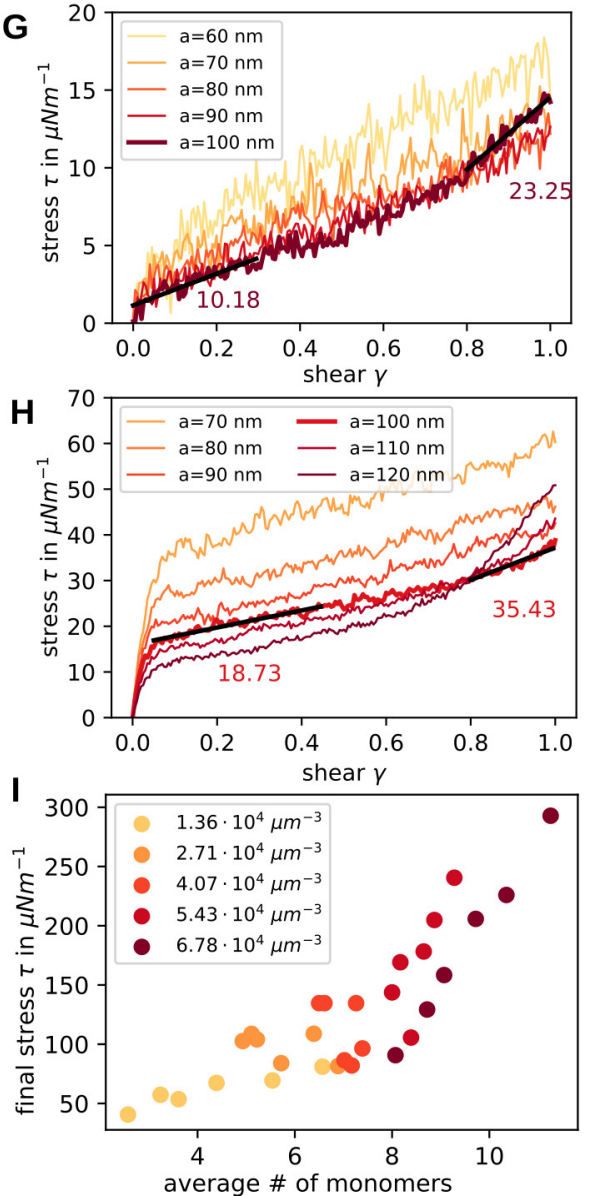

Figure 4. Network shear response. (A) Snapshot of the simulation at shear 1.0 in the $x$-direction. Junctional points are modelled as single actin particles. (B) Same as in a but now the junctions are modelled as full actin filaments. (C) Examples of actin filaments at different lengths within the network. (D-H) Stress is plotted against shear extracted from simulations with a shear rate of $3 \cdot 10^{5} \mathrm{~s}^{-1}$. Each line corresponds to the average of 10 simulations. In $\mathbf{D}$ and $\mathbf{G}$ single particles are used as actin junctions whereas in $\mathbf{E}$ and $\mathbf{H}$ the proper actin filaments are implemented. In $\mathbf{D}$ and $\mathbf{E}$ the spectrin filaments are modelled without angle potentials and the anchoring sites are free to diffuse in the bilayer plane. In $\mathbf{G}$ and $\mathbf{H}$ the spectrin angle potential constant has a strength of $4.28 \mathrm{~kJ} \mathrm{~mol}^{-1}$ and the anchoring sites possess a reduced diffusion due to anchoring in the bilayer. (F)The stress response to shearing is plotted for different average filament lengths as indicated in the legend. Sheared networks are taken from all different concentrations and time points of simulations with capping proteins. (I) The final stress from $\mathrm{C}$ is now plotted against the average filament length with the colours indicating the different initial concentrations as shown in the legend. 
hexagonal configuration, such that the actin distances show a broad distribution [52]. To mimic this, we move the actin particles away from their lattice site by a random displacement (see S3 Fig). In this case we find a higher shear modulus and a stronger strain hardening behaviour in comparison to Figure 4D. This agrees with the analytical calculation showing that random networks are stiffer [52]. This can be explained by the contribution of the strongly elongated spectrin filaments which exhibit strain-hardening properties.

Additionally, we investigated the networks with different actin filament lengths distributions, as analysed in Figure 3D and E, and also applied a shear. In Figure 4C the different possible outcomes are shown. The first panel shows a normal length actin filament, the second one shows completely detached short actin filaments, in the third panel the spectrin filaments are still attached to the very short actin and in the last panel abnormally long actin filaments are shown. Each sheared network is categorised in terms of the average actin filament length and the stress response is plotted in Figure $4 \mathrm{~F}$. As the colour of the curves varies form light to dark green, the average filament length grows. We see that the shear modulus and the initial jump increase with average filament length. To explore this in more detail, the final stress value at shear one is plotted in Figure 4I against the average filament length and the colours show the initial concentration of the simulation that the sheared network configuration was taken from. The higher stress value for longer actin filaments can be confirmed here, where there seem to be two regimes. For average filament lengths of less than seven monomers the fitted slope of stress against length is considerably smaller than for networks with average lengths longer than seven monomers.

\section{Cytoskeletal remodelling by KAHRP}

Using our detailed model of the RBC cytoskeleton, we now are in a position to simulate the effect of KAHRP-binding during a malaria-infection. We introduce particles to the cytoskeletal system that possess the known binding properties of KAHRP, specifically the binding to other KAHRP particles, subunits of the spectrin filaments, ankyrin and actin protofilaments (as explained in the Materials and methods). Because simulations with many interactions are very time consuming, a small membrane patch

$\left(140 \times 242.48 \mathrm{~nm}^{2}\right)$ with periodic boundary conditions and eight actin filaments is set up. The KAHRP particles initially exhibit a uniform random distribution and the lattice constant of the lattice is chosen to be $a=88 \mathrm{~nm}$. Since KAHRP assembly is much faster than the actin dynamics, the effects of actin polymerizaiton on KAHRP assembly are negligible and hence we do not speed up the actin dynamics as in the last section.

In our simulations we varied the relative interaction strength of KAHRP with its different binding partners, the interaction strength itself, the actin filament length and the KAHRP concentration (simulation snapshots in Figure 5A and corresponding videos in S3-S6 Movie). In each case, we quantified the resulting KAHRP cluster. A cluster is defined as all particles whose centres are separated less than the diameter of a KAHRP particle plus a tolerance of $1.5 \mathrm{~nm}$ to the next KAHRP particle and examples are marked with circles in Figure 5A. We distinguish between free floating clusters, clusters attached to the ankyrin binding sites (black circles) and clusters at the actin filaments (red circle). A cluster counts as attached if one of the cluster particles has a gap of less than $1.5 \mathrm{~nm}$ to one of the KAHRP-associating cytoskeletal components.

Our first finding was that when the interaction strength $\epsilon$ is too small compared to the thermal energy, most KAHRP particles will stay separated and detached from the cytoskeleton. In simulations with all interaction energies equal, we found that there is a sharp transition between mostly no clusters present below $\epsilon=2 \mathrm{k}_{\mathrm{B}} \mathrm{T}$ and similarly sized large clusters for $\epsilon \geq 2 \mathrm{k}_{\mathrm{B}} \mathrm{T}$ (compare S4 Fig). These results essentially demonstrate that the energy of clustering competes with the entropy of diffusion in our dynamical 

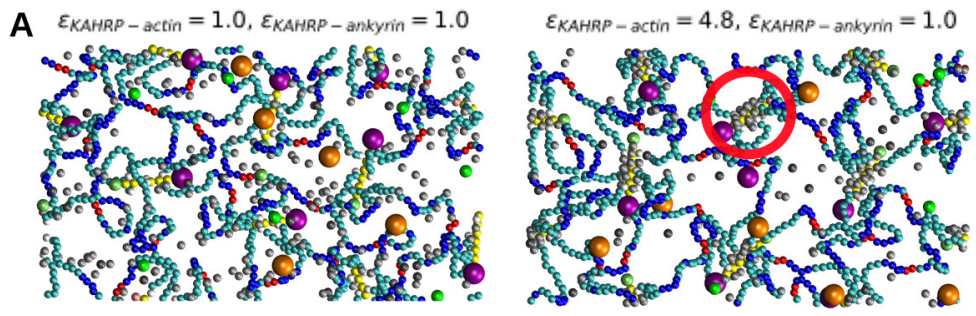

spectrin with KAHRP binding sites in blue and ankyrin binding site in red

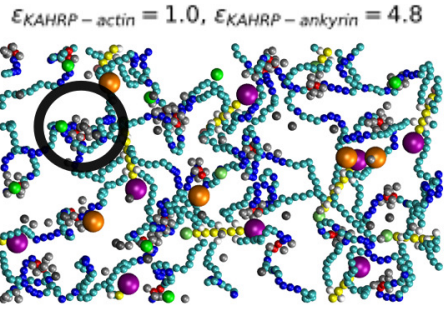

$\varepsilon_{K A H R P-a c t i n}=4.8, \varepsilon_{K A H R P-\text { ankyrin }}=4.8$

junction with capping proteins adducin and tropomodulin
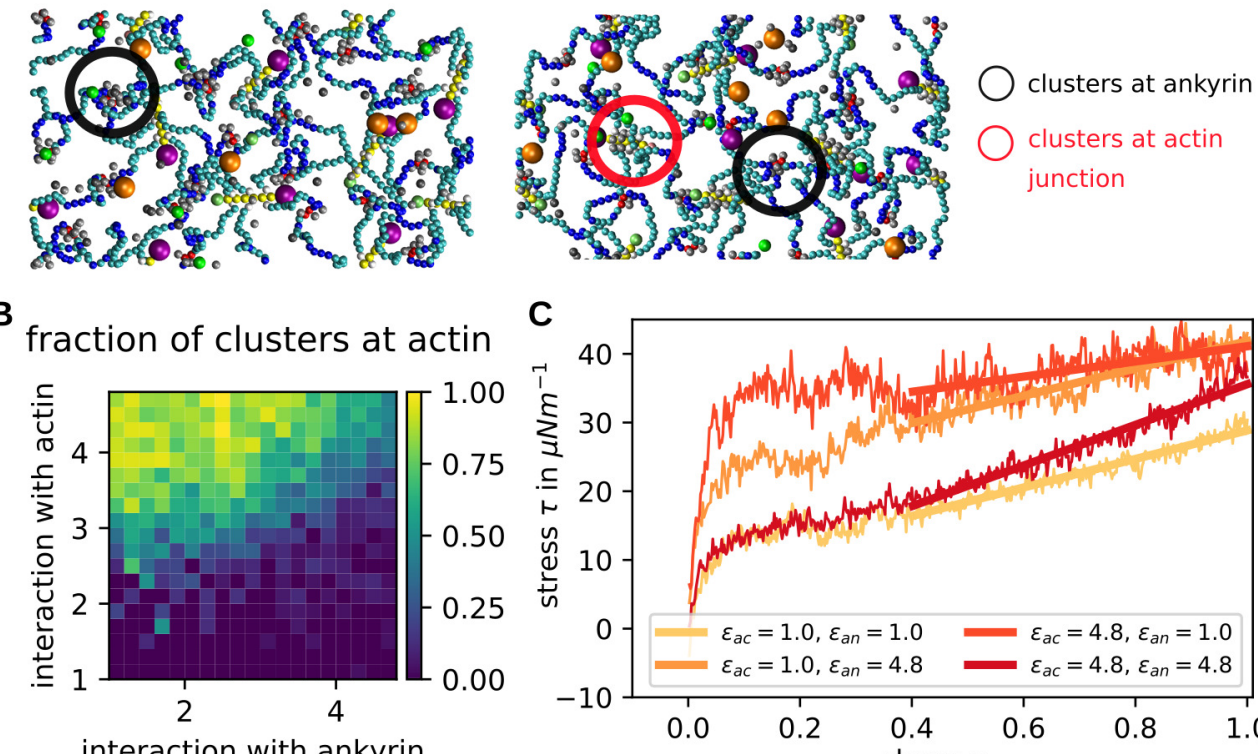

D interaction with ankyrin
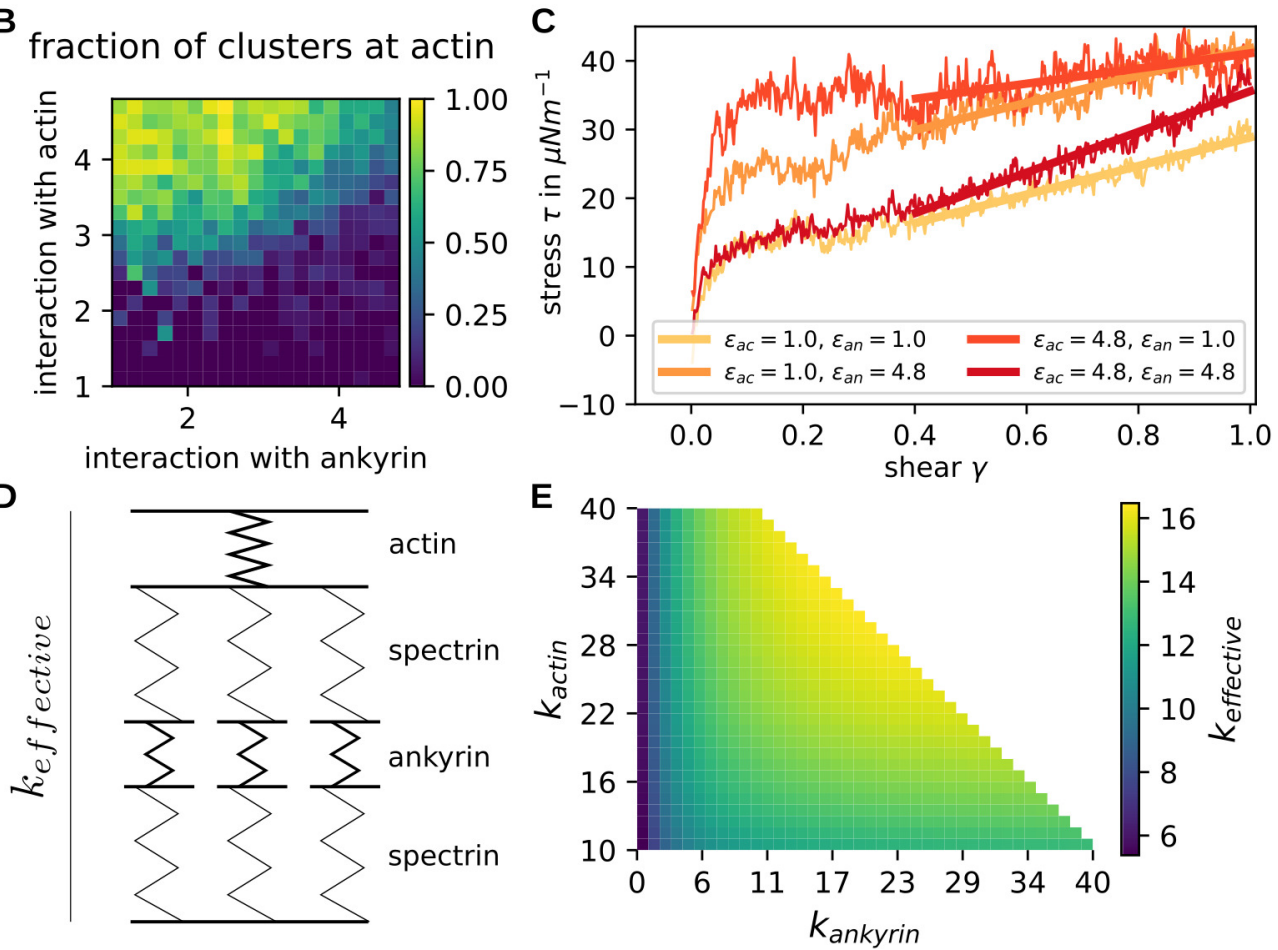

Figure 5. Effect of KAHRP clusters in actin-spectrin network. (A) Snapshots of equilibrated networks for the interaction energies indicated above each image. KAHRP clusters are indicated by circles. (B) Fraction of clusters that are located at actin filaments in contrast to other cytoskeletal subunits. The value is calculated for different interaction energies with actin and ankyrin. (C) Shear response of KAHRP containing networks for different KAHRP positioning. (D) A simple spring model was set up to understand the shear-reasponse of the KAHRP-containing cytoskeletal networks. (E) The effective spring constant is plotted according to our spring model. 
simulation.

Next we varied the interaction energy between KAHRP and actin $\left(\epsilon_{K A H R P-a c t i n}\right)$ and the energy between KAHRP and ankyrin $\left(\epsilon_{K A H R P-a n k y r i n}\right)$, each between 1.0 and $4.8 k_{B} T$. At the same time we fix the interaction energy with KAHRP itself and the spectrin beads at $\epsilon_{K A H R P-K A H R P}=\epsilon_{K A H R P-\text { spectrin }}=1.0 k_{B} T$. For each parameter set we can then extract the properties of KAHRP clusters. Specifically we can calculate the fraction of all KAHRP clusters that are positioned at actin filaments (Figure 5B). The data corresponds to the final configuration of a $200 \mu \mathrm{s}$ simulation. We confirmed that this is the equilibrium configuration by examining the potential energy of the system (not shown here). It can also be seen by looking at the time evolution of the fractions and sizes (shown in the S4 Fig). Snapshots for selected parameters, corresponding to the four diagram corners in Figure 5B are shown in Figure 5A. We see that the interaction with actin needs to be larger than the interaction with ankyrin to reach a high fraction of actin associated clusters (top left corner in Figure 5B). If the interaction is equally strong but large enough, we see a mixed distribution of clusters. Note that no clusters develop if both parameters very small. The size of the clusters can be varied by changing KAHRP concentration (S5 Fig).

To examine the effect of the emerging KAHRP clusters on the mechanical properties of the network, we took the final configurations from different parameter choices discussed in the last paragraph and applied a shear to the network. To do so, the periodic boundary conditions were removed and the network repeated nine times to obtain a larger patch and hence better statistics. Shearing was done at a rate of $3 \cdot 10^{5} \mathrm{~s}^{-1}$. Specifically, we sheared networks with the four extreme cases of KAHRP cluster formation corresponding to the snapshots in Figure 5A: no clusters

$\left(\epsilon_{K A H R P-a c t i n}=1.0 k_{B} T\right.$ and $\left.\epsilon_{K A H R P-a n k y r i n}=1.0 k_{B} T\right)$, mostly ankyrin associated clusters $\left(\epsilon_{K A H R P-a c t i n}=1.0 k_{B} T\right.$ and $\left.\epsilon_{K A H R P-\text { ankyrin }}=4.8 k_{B} T\right)$, mostly actin associated clusters $\left(\epsilon_{K A H R P-a c t i n}=4.8 k_{B} T\right.$ and $\left.\epsilon_{K A H R P-a n k y r i n}=1.0 k_{B} T\right)$ and clusters at both junctions $\left(\epsilon_{K A H R P-a c t i n}=4.8 k_{B} T\right.$ and $\left.\epsilon_{K A H R P-\text { ankyrin }}=4.8 k_{B} T\right)$. The different stress responses are plotted in Figure $5 \mathrm{C}$ and one clearly sees that for clusters at both junctions only the slope is increased and hence the shear modulus, whereas the initial step is higher for the formation of clusters only at one junction type, with the step being the largest for actin-associated clusters. Besides the step the shear modulus is higher for the ankyrin associated clusters.

Previously we saw that longer actin filaments lead to an increased initial step. Therefore, the clustering of KAHRP at one junction type, which leads to relatively large junction points in the network, results in a higher initial step. This effect is larger for actin associated clusters, since the actin forms a larger junction from the start. It seems like networks with clusters at both junction types do not show this effect. This could be explained by the fact that the simulations are run at constant KAHRP concentrations and thus the resulting clusters are smaller because they are distributed over different binding sites. Although the initial jump is smaller, the slope at larger shear is larger. In order to understand this aspect better, we used a simple spring model as shown in Figure 5D which uses different springs for actin/ankyrin junctions and spectrin filaments. The spectrin springs are arranged in parallel to mimic the attachment of several spectrins to one actin junction. The resulting effective spring constant of the simple model was calculated for different actin and ankyrin spring constants and plotted in Figure $5 \mathrm{E}$ with the spectrin spring constant fixed at 5 in non-dimensionalised units. From this plot we see that we expect the largest spring constant and hence shear modulus for a stiffening of both junctions at the same time, as seen in the simulations of Figure $5 \mathrm{C}$. When keeping $k_{\text {ankyrin }}$ fixed at a low values, which corresponds to clusters at only the actin junctions, we expect nearly no increase in shear modulus as seen in Figure $5 \mathrm{E}$ on the left side for a change along the $\mathrm{y}$-axis. In our shear experiments the 
slope stayed very low as expected here. Finally, for the case of ankyrin associated clusters, we see that the effective spring constant does change when going along the $\mathrm{x}$-axis of Figure 5E. Note that we start the plot at an actin spring constant of 10 in order to take into account that the actin junction is the stiffest element of the network without KAHRP present. This explains the observed higher shear modulus for ankyrin associated clusters in contrast to actin associated clusters.

\section{Pair cross-correlation analysis}

Finally we used pair cross-correlation (PCC) analysis to connect our simulation results with the experimental results from our very recent two-color super-resolution microscopy study of dynamic KAHRP localization within the iRBC cytoskeleton [34]. Figure 6A shows a sample image of fluorescently labeled KAHRP and ankyrin in an exposed RBC membrane skeleton and Figures $6 \mathrm{~B}$ and $\mathrm{C}$ show the resulting PCCs, which can be interpreted as the probability of finding two signals at a given radial distance. For co-localizing signals, the highest PCC is observed at a zero distance and for large separations between signals, the highest PCC shifts to a finite distance.

Figure $6 \mathrm{~B}$ shows that the PCC profile between KAHRP and ankyrin shifts from a maximum value at zero up to 16 hours to a flat profile at 20 hours and eventually to a maximum value at a finite distance $(\approx 110 \mathrm{~nm})$ at $28-36$ hours post malaria infection. In contrast, the PCC between KAHRP and the N-terminus of spectrin shows the maximum value at zero throughout, as shown in Figure 6C. This suggests that during the malaria infection, KAHRP relocates and moves away from the ankyrin complexes and towards the actin complexes.

To compute PCCs from our simulations, we generated a spatial distribution profile for each protein as described in the Materials and methods. Figure 6D shows the generated images for KAHRP in red and ankyrin in green colour. We first note that these images should not be compared directly to the experimental images in Figure 6A, because the scale is much smaller and the regularity is much higher due to the assumed hexagonal lattice in the computer simulations. Nevertheless we can calculate PCCs from them which can be compared to the experimental data. In Figure $6 \mathrm{E}$ and $\mathrm{F}$ we show the calculated PCCs for KAHRP with ankyrin and for KAHRP with the

N-terminus of spectrin located at actin junction, respectively, for different values of the binding energy between KAHRP and actin. The PCC of KAHRP with ankyrin decreases with the increase in its binding energy with actin, and the maximum PCC shifts from zero distance to a finite distance. This distance corresponds to the separation between actin and ankyrin junctions $(\approx 55 \mathrm{~nm})$ in our simulated RBC-networks. However, the experimental PCC (shown in Figure 6B) shows a larger separation distance between the two junctions. This suggests that the RBC networks in the experiments are highly stretched. In contrast to the PCC with ankyrin, the highest PCC of KAHRP with the N-terminus of spectrin remains at a zero distance. Further, for strong KAHRP-actin binding, we observe a second peak at larger distances due to the KAHRP's correlation with N-terminus of spectrin present at the next nearest neighbour site of the RBC network.

Next, we varied binding energy between KAHRP and actin and KAHRP and ankyrin to systematically identify the regions in the parameter space where such behavior exists. In Figure 6G, we plot the PCC at zero distance for different binding energy values and mark the cases with finite distance peak with white dots. For short actin filaments, KAHRP-ankyrin PCC shows a maximum at zero distance in large regions of the parameter space, except in areas with very high KAHRP-actin binding. In contrast, the KAHRP and N-terminus of spectrin PCC shows the maximum at a finite distance for high KAHRP-ankyrin and low KAHRP-actin binding energy. This is because KAHRP preferentially binds to the ankyrin junction, thus not at the actin 
A

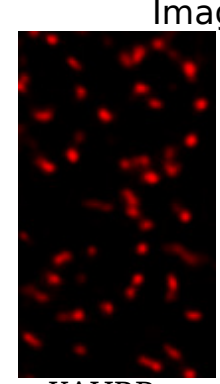

D KAHRP
Images from experiments

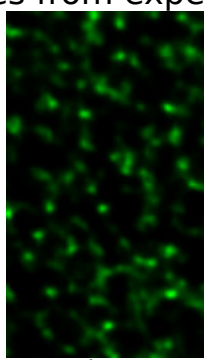

Ankyrin Images from simulations

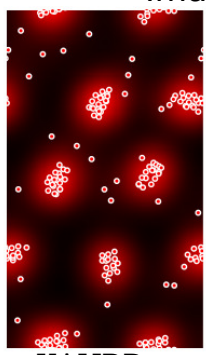

KAHRP

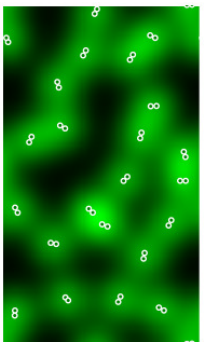

Ankyrin

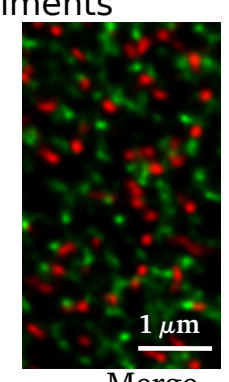

B

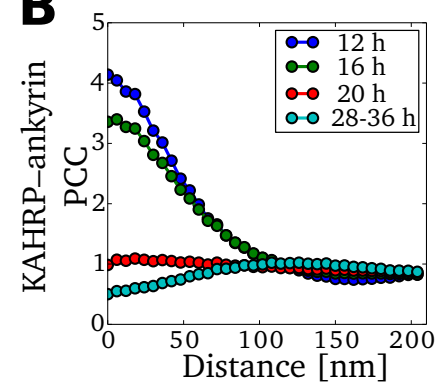

$\mathbf{E}$
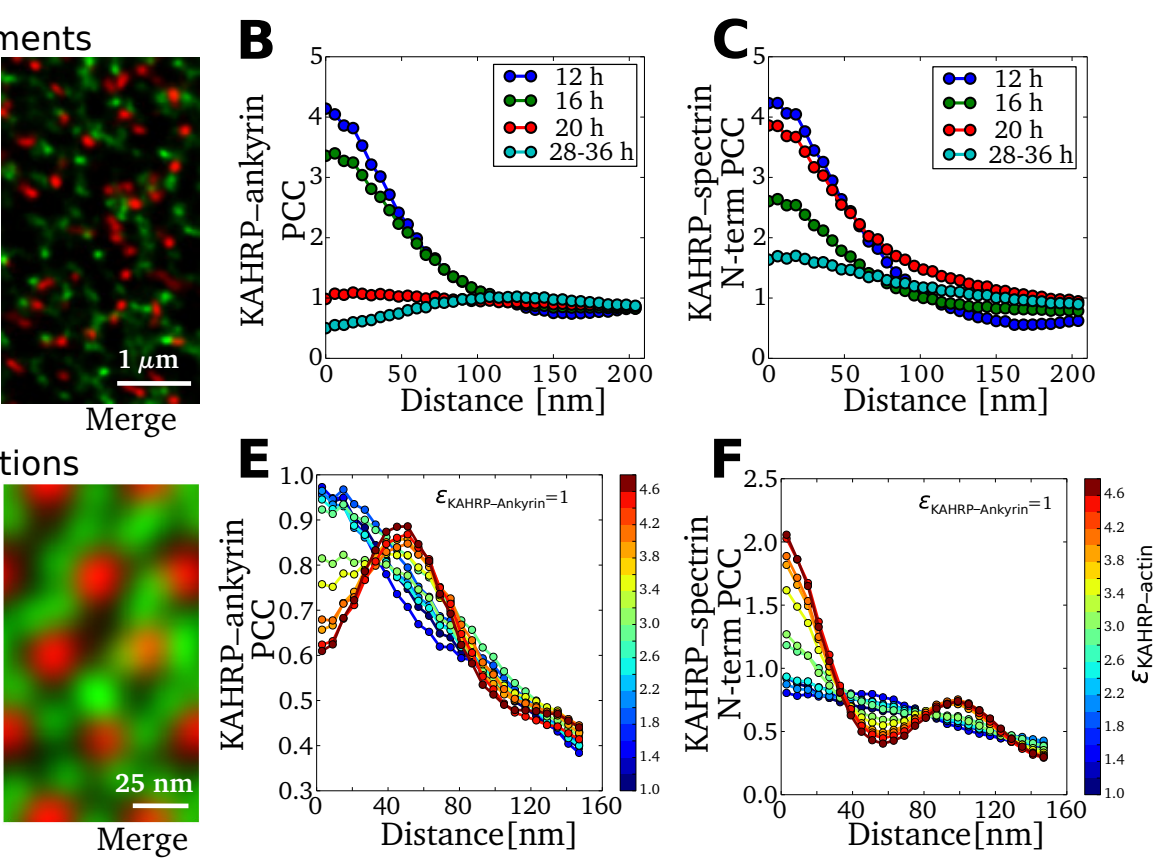

$\mathbf{G}$

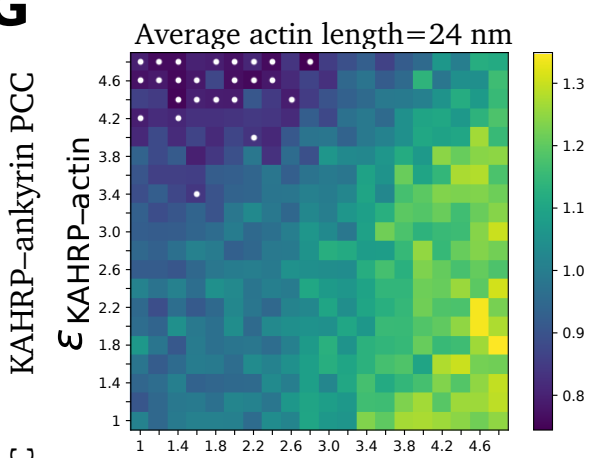

H Average actin length $=36 \mathrm{~nm}$

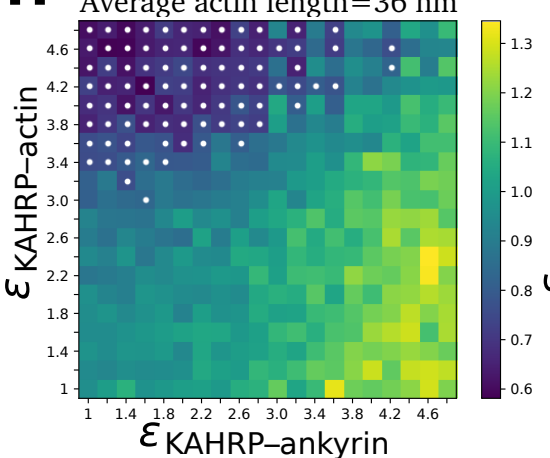

I Average actin length $=48 \mathrm{~nm}$

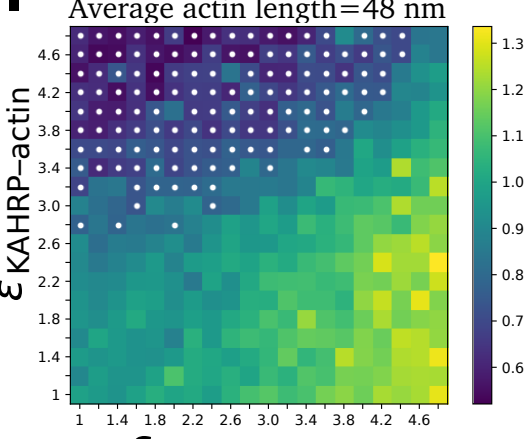

$\varepsilon_{\text {KAHRP-ankyrin }}$
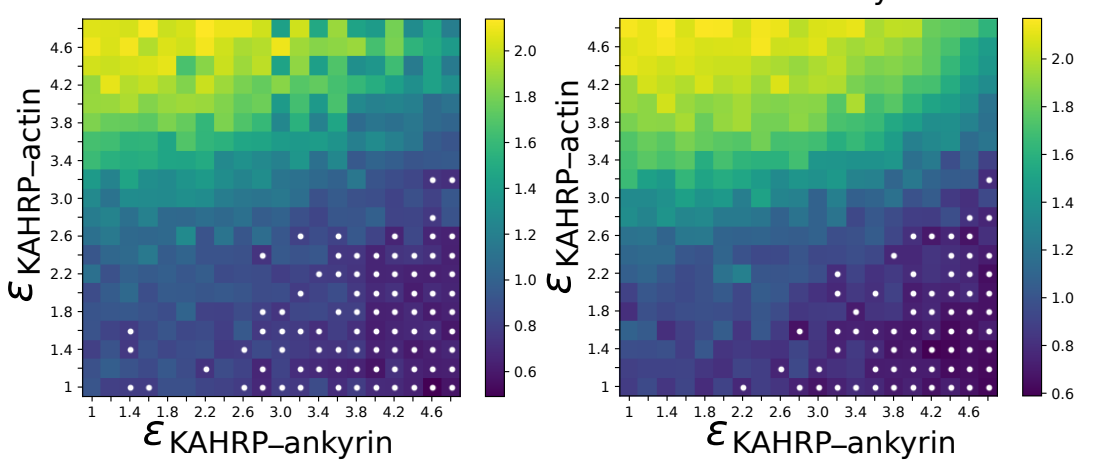

Figure 6. Comparison of experimental and simulated pair cross-correlations. (A) STED images of an exposed RBC membrane at the trophozoite stage (28 - 36 hours post malaria infection). Red and green fluorescence signals correspond to KAHRP and ankyrin sites on the RBC membrane. (B) Pair cross-correlation (PCC) between KAHRP and ankyrin computed using the two-color images obtained at different hours post malaria infection. (C) PCC between KAHRP and N-terminus of spectrin. Images and PCC values in (A,B) are taken from [34]. (D) KAHRP (red) and ankyrin (green) fluorescence signal is constructed from location points obtained from simulations (overlaid white points). (E) PCC between KAHRP and ankyrin is computed from two-color images generated from simulations for different binding energy between KAHRP and actin. (F) Same as (E) for the KAHRP and N-terminus of spectrin PCC. (G) The map shows PCC values at zero distance for KAHRP-actin pairs (top panel) and KAHRP-ankyrin pairs (bottom panel) at different binding rates between KAHRP and ankyrin/actin sites for average actin filament length $24 \mathrm{~nm}$. The white dots mark the cases where a peak is observed at a finite distance. (H) and (I) Same as (G) for average actin filament length $36 \mathrm{~nm}$ and $48 \mathrm{~nm}$ respectively. 
junction where the N-terminus of spectrin is located. At an increased actin length (Figure $6 \mathrm{H}$ for $36 \mathrm{~nm}$ and Figure 6I for $48 \mathrm{~nm}$ ), we observe increased regions in the parameter space where the KAHRP-ankyrin PCC shows a maximum at a finite distance for high KAHRP-actin and low KAHRP-ankyrin binding energy. These results suggest that the temporal change in KAHRP-ankyrin PCC during the malaria infection can be explained both by differences in the binding affinity between KAHRP and actin or by changes in actin filament length. We also investigate the effect of KAHRP concentration on the pair cross-correlation values. The increase in KAHRP concentration decreases the region where the PCC maximum at a finite distance is observed (S6 Fig).

\section{Discussion}

In order to theoretically analyse the dramatic transformation of the RBC cytoskeleton during a malaria infection, here we have developed a particle-based simulation framework that incorporates both the structure and dynamics of actin filaments and the different known binding sites of KAHRP, which is the most important parasite-derived factor for this transformation. Our coarse-grained Brownian dynamics simulations allow for sufficiently long and large simulations to measure the effective cellular shear modulus as a function of the key molecular processes. The shear modulus in turn is the central quantity to predict how iRBCs will move in the blood flow and through the interendothelial slits of the spleen. Our work is scale-bridging in the sense that it connects the spatial coordination of essential molecular processes, that are increasingly investigated with super-resolution microscopy [17,30,34], with cellular properties like the shear modulus, which has been measured before with different experimental techniques [22,23].

Our first main result is the observation that including explicit actin filaments into the simulations leads to two regimes during shearing, namely a fast and stiff response corresponding to their reorientation and a slow and less stiff response corresponding to the reorganization of the spectrin network. This latter response shows strain stiffening, as expected, and agrees quantitatively with results from similar coarse-grained computer simulations in which the actin protofilaments have been modelled as particles $[33,35,36]$. These results also agree well with values for the shear modulus extracted from experiments with optical tweezers [22] or diffraction phase microscopy [23]. Regarding the identification of a fast and stiff initial response, we note that it seems challenging to demonstrate this effect in experiments, because it would require very high time resolution and good preparation of the initial state. Yet we believe that this is an interesting new effect that might play a role in the physiological context. In the future, a more detailed investigation should also include the possibilities of actin protofilaments flipping out of the plane of the membrane [53] and of unfolding of spectrin

repeats $[54,55]$. Both of these processes could yield extra length that would smear out the effect of the rapid ordering of the actin protofilaments. We finally note that whole cell simulations with molecular details are required to decide how this effect is averaged over the complete area of the RBC [36].

Our simulations not only modeled for the first time the actin component as explicit filaments, they also included their dynamical nature, allowing for them to shrink or grow. We found that by introducing length-dependent capping rates with dissociation constants matching experimental observations, filaments could be held stable over a longer time period than without capping proteins present. In order to improve the model in this respect, the rules for tropomyosin attachment should be refined. By testing different G-actin concentrations, filament networks of various average filament lengths were produced and their response to shear could be determined. We found that shorter filaments lead to a reduced stress because the network looses connection points, 
suggesting that the actin mining by the parasite should reduce the shear modulus if no other processes were present.

The most important remodelling process triggered by the parasite is the clustering of the protein KAHRP, which has known binding sites to different components of the membrane skeleton. By introducing new particles into the computer simulations that possess the properties of KAHRP molecules, the KAHRP cluster formation could be analysed in detail, yielding a different cluster positioning within the cytoskeleton depending on the relation between the interaction strengths of KAHRP with its binding partners. In general, we found that KAHRP clustering, which eventually corresponds to knob formation, leads to an increase of the shear modulus, in agreement with earlier computer simulations that focused on the mature knobs [33]. By varying the different interaction strengths (which could also be interpreted as different values of $K_{D}$ ), we could predict the effect on the shear modulus for different scenarios of KAHRP clustering. In principle, the recently observed KAHRP relocalization from ankyrin to actin complexes could result from different effects, including changes in binding affinity due to phosphorylation, increase in concentration and structural changes to the target sites (in particular the remodelled actin junctional complexes). Our simulations showed that the first mechanism is the most likely candidate, because its effect resembles the experimentally observed dynamics. Our conclusion is in agreement with experimental observations that during the intra-erythrocytic cycle the phosphorylation and/or acetylation pattern of KAHRP are changing $[56,57]$. While the effect of KAHRP concentration seemed to be small in the simulations, dynamic changes of actin filament length did play some role, as they could increase the number of binding sites at the knobs.

In order to compare simulations and experiments, we used a pair cross-correlation (PCC) analysis. We found that indeed the experimentally observed time course can be recapitulated by increases in the binding affinity between KAHRP and actin. Because our computer simulations work on a better resolved scale and assume a regular lattice, the corresponding PCCs show more structure than the experimentally measured ones, similar to earlier work on healthy RBCs [17], but the qualitative agreement is good enough to conclude that dynamical changes in KAHRP binding affinities are the most likely mechanism for the observed spatial changes. In the future, dedicated experiments are required to identify the corresponding kinases and phosphorylation sites.

In summary, our newly developed computer simulations provide an exploratory tool to investigate the different points of attacks that the malaria parasite might use to remodel the membrane skeleton of iRBCs to achieve favorable flow and adhesion behaviours in the vasculature. Here we focused on spatial processes on the molecular scale that directly translate into the mechanical properties relevant for cell movement in hydrodynamic flow. Our finding that KAHRP affinity might be the central feature of interest suggests that also other more biochemical processes might play a role, including regulation of actin capping and oxidative stress. Before these important processes can be included here, more experimental evidence is required to guide such a modelling approach. We finally note that future work should also become more three-dimensional. Although our simulations are three-dimensional in regard to the spectrin network and reproduce well the known thickness of this layer, they did not consider

three-dimensional changes for the actin protofilaments [53], the formation of the long actin filaments connected to the Maurer's clefts [19] or the spiral scaffold underlying the knobs $[29,30,34]$. As a long-time perspective, it would be very desirable to develop also a spatial model for these more three-dimensional processes. 


\section{Supporting information}

\section{S1 File. Details on the modelling procedure.}

S1 Table. Parameters for the repulsion potentials. The value for spectrin is chosen to match previous simulations in Ref. [35].

S2 Table. Summary of actin dynamics rates. Rates and concentrations relevant for the actin dynamics are collected here. The real value is given with its reference where applicable and the value scaled in order to produce observable actin dynamics within an accessable time period. The scaling factor $\mathrm{b}$ was set to $10^{5}$.

S1 Fig. Network configuration. (A) Definition of actin filament angles in space. Both angles can vary between 0 and $90^{\circ}$ as the filament polarity does not matter. (B) Distribution of the out of plane angle $\phi$ at the initial and final time point of the simulation. The data from 10 independent runs of a network with 46 actin filaments each is used. (C) Same as in b but for the orientation within the plane of the bilayer quantified with angle $\theta$. (D) Side view of a simulation snapshot with a confining potential for the actin filaments near the bottom of the simulation box. Actin filaments are shown in yellow and spectrin filaments in cyan and blue. Diffusing monomers are important for the actin polymerisation. (E) Distribution of different particle types given as distance from the bottom simulation boundary mimicking the lipid bilayer. Actin filaments and spectrin filaments are considered separately.

S2 Fig. Shear rate effects. Effect of different shear rates on the stress in the modelled network is shown for different lattice constants. (A) The shear rate was set to $1.5 \cdot 10^{5} \mathrm{~s}^{-1}$. (B) The shear rate was set to $5.0 \cdot 10^{4} \mathrm{~s}^{-1}$. C) The shear rate was set to $1.7 \cdot 10^{4} \mathrm{~s}^{-1}$.

S3 Fig. Effects of random displacement on shear response. For these shear simulations the position of the actin particles was randomly displaced from their hexagonal lattice site. The extracted stress is shown here for different lattice constants.

S4 Fig. Formation of actin-associated clusters. (A-C) The fraction of clusters near actin filaments is plotted over time and distinct conditions as explained hereafter. (D-F) Average size of cytoskeleton attached clusters is plotted for the same conditions. In (A) and (D) the relative strength between the KAHRP-actin and the KAHRP-spectrin interaction is varied. In (B) and (E) the interaction strength is varied and in (D) and (F) the KAHRP concentration. For each of the three columns the other two parameters are kept fixed at the value indicated by the red horizontal line. Each data point corresponds to the average of three simulations.

S5 Fig. Effects of KAHRP concentration on cluster size. The map shows the average size of different KAHRP clusters, i.e., free, actin-associated, and ankyrin-associated for different binding energy between KAHRP and ankyrin/actin junctions and for different concentrations of KAHRP.

S6 Fig. Effect of KAHRP concentration on pair cross-correlation. The map shows PCC at zero distance for KAHRP-actin pairs (top panel) and KAHRP-ankyrin pairs (bottom panel) for different KAHRP concentrations shown in Figure S4 Fig. 
Table S1. S1 Table

\begin{tabular}{|l|c|c|c|c|}
\hline$k_{\text {repulsion }}$ in $\frac{\mathrm{kJ}}{\mathrm{mol} \mathrm{nm}^{2}}$ & actin & spectrin & adducin & tropomodulin \\
\hline actin & 10 & 38.11 & 10 & 10 \\
spectrin & & 38.11 & 38.11 & 38.11 \\
adducin & & & 10 & 10 \\
tropomodulin & & & & 10 \\
\hline
\end{tabular}

\begin{tabular}{|c|r|r|l|}
\hline Parameter & Real Value & Scaled Value & Source \\
\hline \hline cytosolic G-actin concentration & $0.36 \mu \mathrm{M}$ & $108396 \mathrm{\mu m}^{-3}$ & Gokhin et al. $[16]$ \\
\hline cytosolic tropomodulin concentration & $0.005 \mu \mathrm{M}$ & $1506 \mathrm{\mu m}^{-3}$ & Gokhin et al. $[16]$ \\
\hline cytosolic adducin concentration & not known & $1506 \mathrm{\mu m}^{-3}$ & same as tropomod. \\
\hline \hline macroscopic association rates & \multicolumn{3}{|l|}{} \\
\hline \hline barbed end & $11.6 \mu \mathrm{M}^{-1} \mathrm{~s}^{-1}$ & $3.86 \mathrm{\mu m}^{3} \mathrm{~s}^{-1}$ & Pollard [58] \\
\hline pointed end & $1.3 \mu \mathrm{M}^{-1} \mathrm{~s}^{-1}$ & $0.434 \mathrm{\mu m}^{3} \mathrm{~s}^{-1}$ & Pollard [58] \\
\hline adducin & not known & $2.5 \mathrm{\mu m}^{3} \mathrm{~s}^{-1}$ & match $K_{D}$ \\
\hline tropomodulin & not known & $1.5 \mathrm{\mu m}^{3} \mathrm{~s}^{-1}$ & match $K_{D}$ \\
\hline \hline microscopic association rates & no value & $2.38 \times 10^{7} \mathrm{~s}^{-1}$ & simulation specific \\
\hline \hline barbed end & no value & $5.3 \times 10^{5} \mathrm{~s}^{-1}$ & simulation specific \\
\hline pointed end & no value & $1.11 \times 10^{7} \mathrm{~s}^{-1}$ & simulation specific \\
\hline adducin & no value & $7.18 \times 10^{6} \mathrm{~s}^{-1}$ & simulation specific \\
\hline tropomodulin & \multicolumn{3}{|l}{} \\
\hline \hline dissociation rates & $1.4 \mathrm{~s}-1$ & $1.4 \times 10^{5} \mathrm{~s}^{-1}$ & Pollard [58] \\
\hline barbed end & $0.8 \mathrm{~s}$ & $0.8 \times 10^{5} \mathrm{~s}^{-1}$ & Pollard [58] \\
\hline pointed end & not known & $7.5 \times 10^{4} \mathrm{~s}^{-1}$ & match $K_{D}$ \\
\hline adducin & not known & $4.52 \times 10^{2} \mathrm{~s}^{-1}$ & match $K_{D}$ \\
\hline tropomodulin (with tropomyosin) & not known & $1.35 \times 10^{5} \mathrm{~s}^{-1}$ & match $K_{D}$ \\
\hline tropomodulin (no tropomyosin) & \multicolumn{4}{|l}{}
\end{tabular}

Table S2. S2 Table

S1 Movie. Simulation of the healthy RBC cytoskeleton.

S2 Movie. Movie of RBC cytoskeleton shear simulation.

S3 Movie. Simulation of the RBC cytoskeleton for low KAHRP-actin and KAHRP-ankyrin binding energy.

S4 Movie. Simulation of the RBC cytoskeleton for high KAHRP-actin and low KAHRP-ankyrin binding energy.

S5 Movie. Simulation of the RBC cytoskeleton for low KAHRP-actin and high KAHRP-ankyrin binding energy. 
bioRxiv preprint doi: https://doi.org/10.1101/2021.10.04.462981; this version posted October 4, 2021. The copyright holder for this preprint (which was not certified by peer review) is the author/funder, who has granted bioRxiv a license to display the preprint in perpetuity. It is made available under aCC-BY 4.0 International license.

A

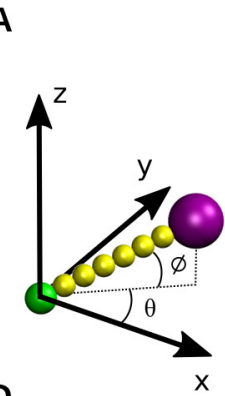

D

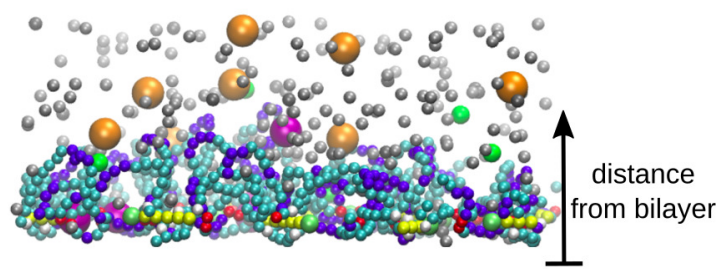

Figure S1. S1 Fig

B
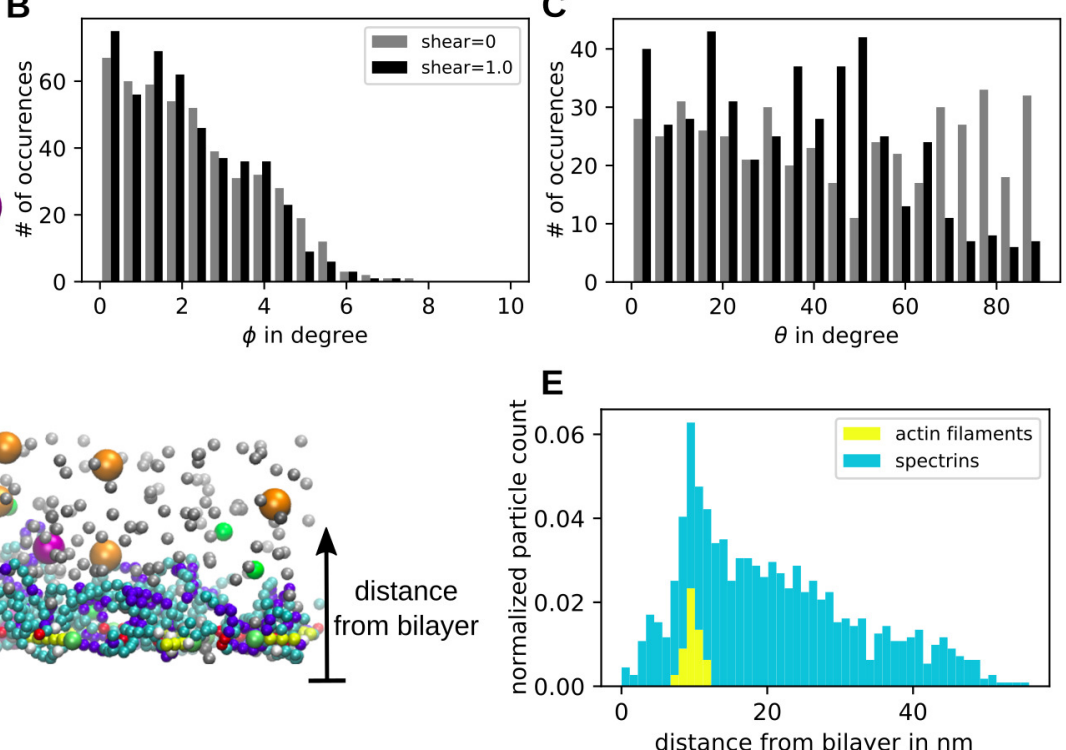

C

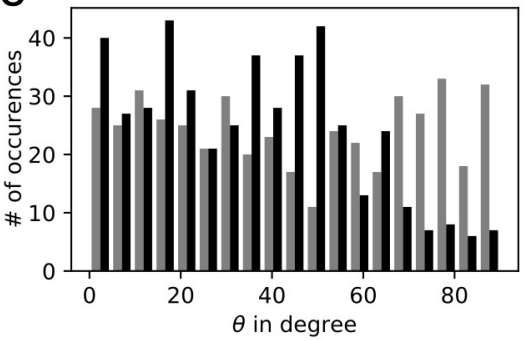

distance from bilayer in $\mathrm{nm}$ 
bioRxiv preprint doi: https://doi. org/10.1101/2021.10.04.462981· this version posted October 4, 2021. The copyright holder for this preprint (which was not certified by peer review) is the author/funder, who has granted bioRxiv a license to display the preprint in perpetuity. It is made available under aCC-BY 4.0 International license.
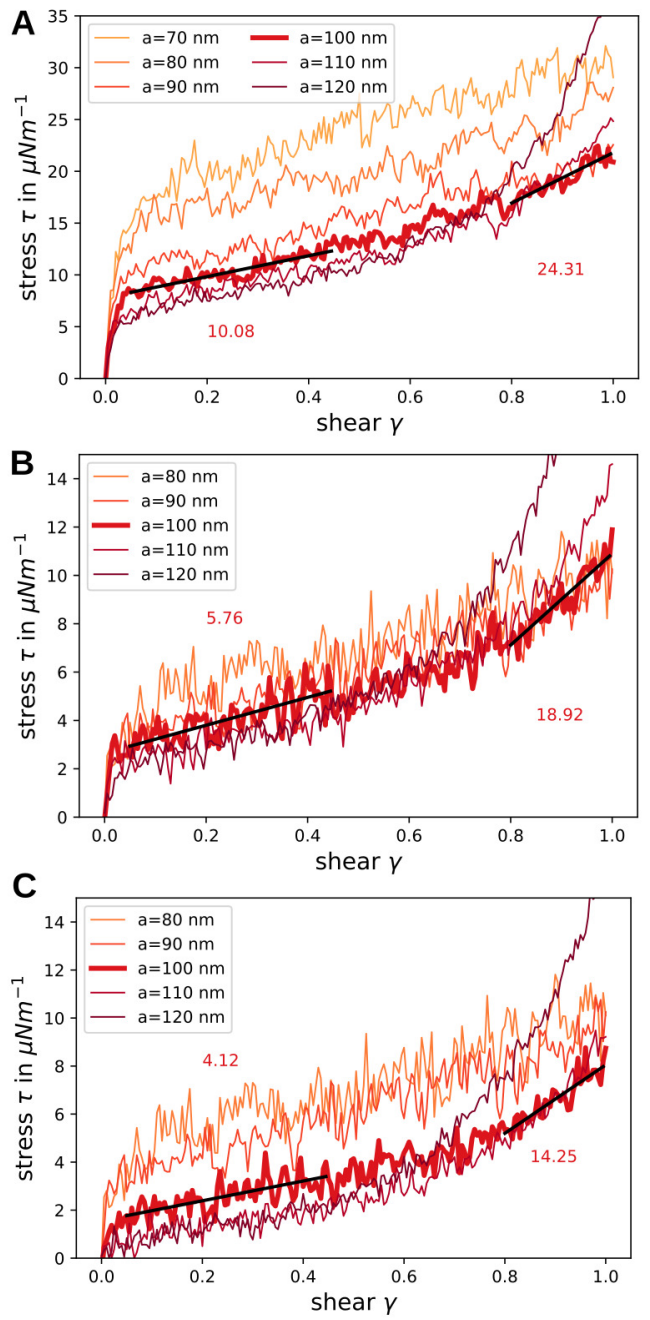

Figure S2. S2 Fig

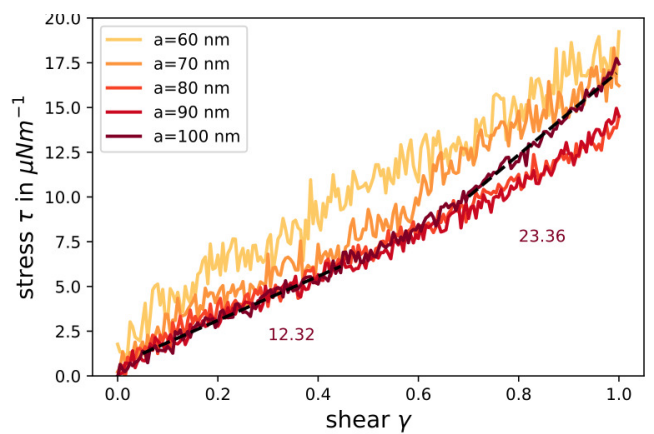

Figure S3. S3 Fig 
bioRxiv preprint doi: https://doi.org/10.1101/2021.10.04.462981; this version posted October 4, 2021. The copyright holder for this preprint (which was not certified by peer review) is the author/funder, who has granted bioRxiv a license to display the preprint in perpetuity. It is made available under aCC-BY 4.0 International license.
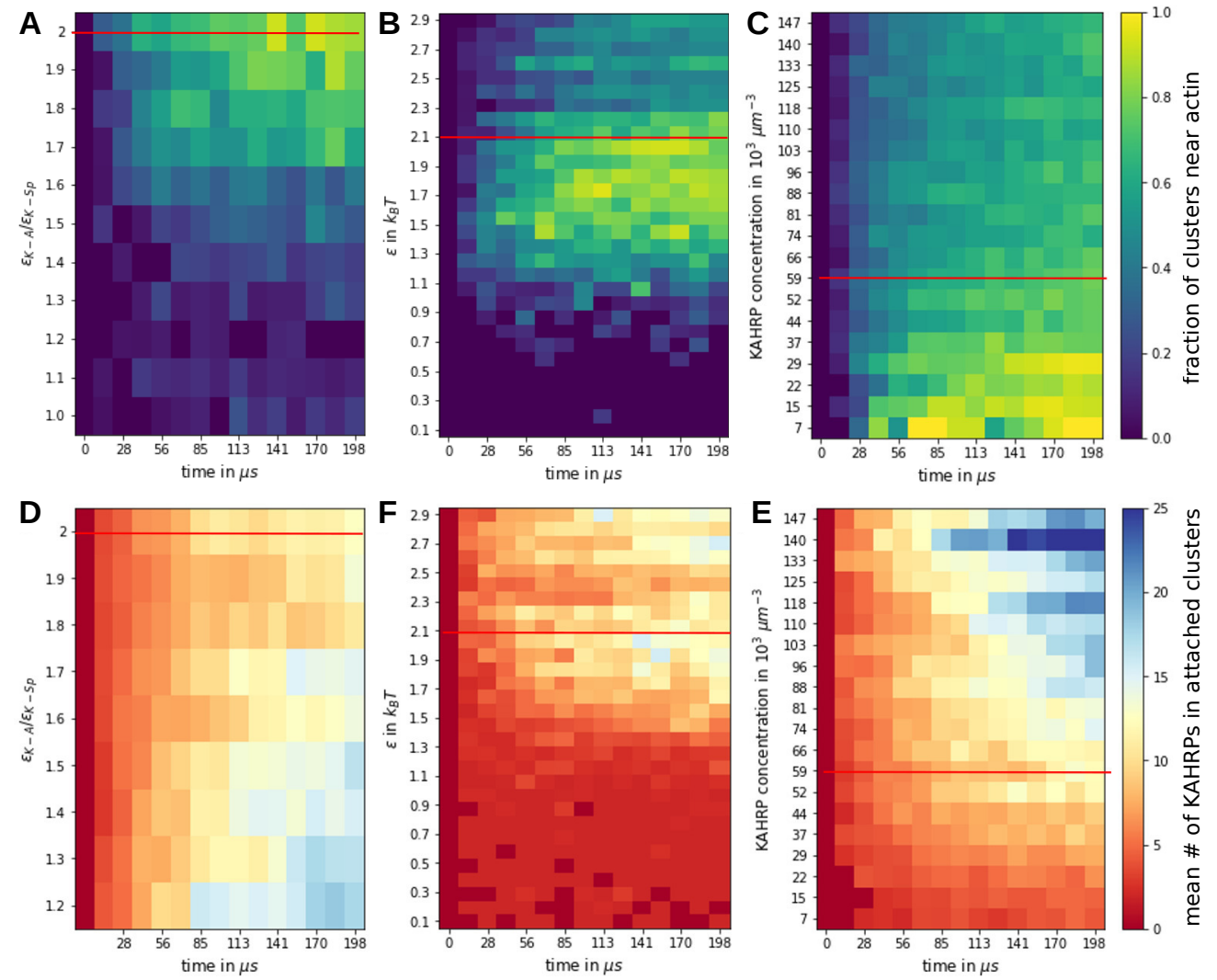

Figure S4. S4 Fig 
bioRxiv preprint doi: https://doi.org/10.1101/2021.10.04.462981; this version posted October 4, 2021. The copyright holder for this preprint (which was not certified by peer review) is the author/funder, who has granted bioRxiv a license to display the preprint in perpetuity. It is made available under aCC-BY 4.0 International license.

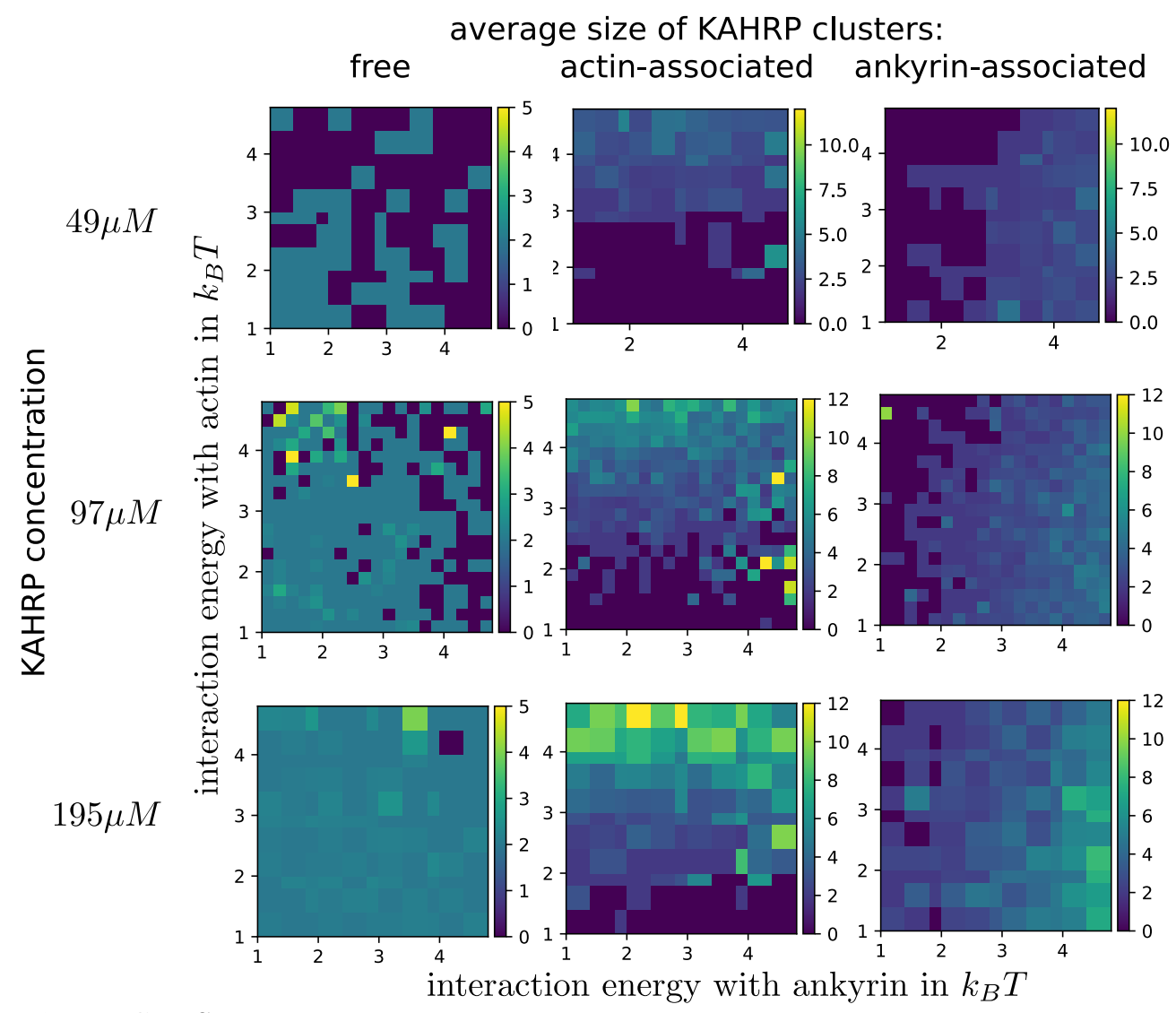

Figure S5. S5 Fig
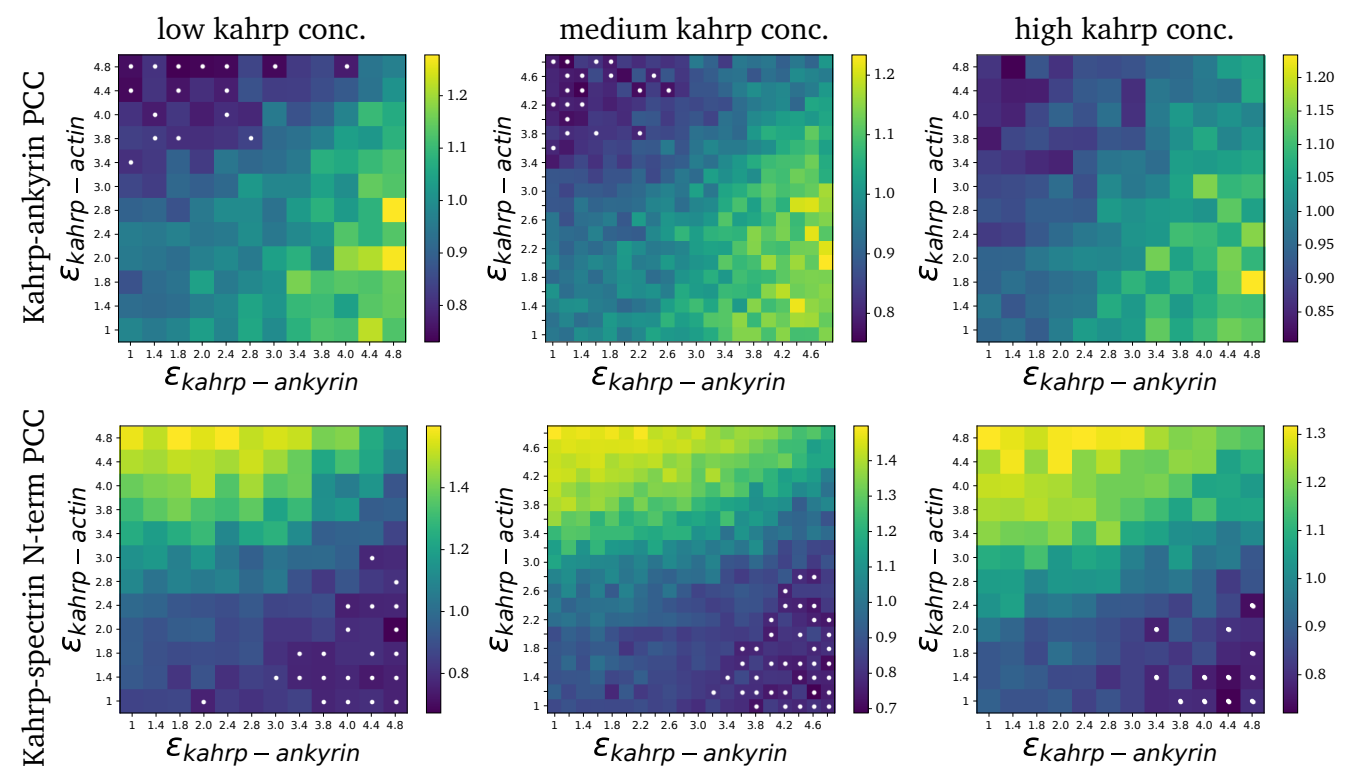

Figure S6. S6 Fig 


\section{Acknowledgments}

This work was funded by the Deutsche Forschungsgemeinschaft (DFG, German Research Foundation) through grants from SFB 1129 to ML and USS (Projektnummer 240245660). JJ was supported by a fellowship from the Heidelberg Graduate School of Mathematical and Computational Methods for the Sciences (HGS MathComp).

\section{References}

1. World malaria report 2020 by the World Health Organization; 2020. Available from: https://www. who.int/publications/i/item/9789240015791.

2. Cowman AF, Healer J, Marapana D, Marsh K. Malaria: Biology and Disease. Cell. 2016;167(3):610-624. doi:10.1016/j.cell.2016.07.055.

3. Warncke JD, Beck HP. Host Cytoskeleton Remodeling throughout the Blood Stages of Plasmodium falciparum. Microbiology and Molecular Biology Reviews. 2019;83(4):e00013-19. doi:10.1128/MMBR.00013-19.

4. Maier AG, Cooke BM, Cowman AF, Tilley L. Malaria parasite proteins that remodel the host erythrocyte. Nature Reviews Microbiology. 2009;7(5):341-354.

5. Mohandas N, Gallagher PG. Red cell membrane: past, present, and future. Blood. 2008;112(10):3939-3948. doi:10.1182/blood-2008-07-161166.

6. Lux SE. Anatomy of the red cell membrane skeleton: unanswered questions. Blood. 2015;127:187-199. doi:10.1182/blood-2014-12-512772.

7. Machnicka B, Czogalla A, Hryniewicz-Jankowska A, Bogusławska DM, Grochowalska R, Heger E, et al. Spectrins: a structural platform for stabilization and activation of membrane channels, receptors and transporters. Biochimica Et Biophysica Acta. 2014;1838(2):620-634. doi:10.1016/j.bbamem.2013.05.002.

8. Stokke BT, Mikkelsen A, Elgsaeter A. Human erythrocyte spectrin dimer intrinsic viscosity: temperature dependence and implications for the molecular basis of the erythrocyte membrane free energy. Biochimica Et Biophysica Acta. 1985;816(1):102-110. doi:10.1016/0005-2736(85)90398-0.

9. Svoboda K, Schmidt CF, Branton D, Block SM. Conformation and elasticity of the isolated red blood cell membrane skeleton. Biophysical Journal. 1992;63(3):784-793.

10. Rief M, Pascual J, Saraste M, Gaub HE. Single molecule force spectroscopy of spectrin repeats: low unfolding forces in helix bundles11Edited by W. Baumeister. Journal of Molecular Biology. 1999;286(2):553-561. doi:10.1006/jmbi.1998.2466.

11. Stokke BT, Mikkelsen A, Elgsaeter A. Spectrin, human erythrocyte shapes, and mechanochemical properties. Biophysical Journal. 1986;49(1):319-327. doi:10.1016/S0006-3495(86)83644-X.

12. Boal DH. Computer simulation of a model network for the erythrocyte cytoskeleton. Biophysical Journal. 1994;67(2):521-529. doi:10.1016/S0006-3495(94)80511-9.

13. Picart C, Dalhaimer P, Discher DE. Actin Protofilament Orientation in Deformation of the Erythrocyte Membrane Skeleton. Biophysical Journal. 2000;79(6):2987-3000. doi:10.1016/S0006-3495(00)76535-0. 
14. Heinrich V, Ritchie K, Mohandas N, Evans E. Elastic thickness compressibilty of the red cell membrane. Biophysical Journal. 2001;81(3):1452-1463.

doi:10.1016/S0006-3495(01)75800-6.

15. W GLH, Wortis M, Mukhopadhyay R. Stomatocyte-discocyte-echinocyte sequence of the human red blood cell: Evidence for the bilayer-couple hypothesis from membrane mechanics. PNAS. 2002;99(26):16766-16769.

doi:10.1073/pnas.202617299.

16. Gokhin DS, Nowak RB, Khoory JA, Piedra Adl, Ghiran IC, Fowler VM. Dynamic actin filaments control the mechanical behavior of the human red blood cell membrane. Molecular Biology of the Cell. 2015;26(9):1699-1710. doi:10.1091/mbc.E14-12-1583.

17. Pan L, Yan R, Li W, Xu K. Super-Resolution Microscopy Reveals the Native Ultrastructure of the Erythrocyte Cytoskeleton. Cell Reports. 2018;22(5):1151-1158. doi:10.1016/j.celrep.2017.12.107.

18. Nowak RB, Alimohamadi H, Pestonjamasp K, Rangamani P, Fowler VM. Nanoscale organization of Actin Filaments in the Red Blood Cell Membrane Skeleton. bioRxiv. 2021; p. 2021.03.07.434292. doi:10.1101/2021.03.07.434292.

19. Cyrklaff M, Sanchez CP, Kilian N, Bisseye C, Simpore J, Frischknecht F, et al. Hemoglobins $\mathrm{S}$ and $\mathrm{C}$ interfere with actin remodeling in Plasmodium falciparum-infected erythrocytes. Science. 2011;334(6060):1283-1286.

20. Gokhin DS, Fowler VM. Feisty filaments: actin dynamics in the red blood cell membrane skeleton. Current opinion in hematology. 2016;23(3):206-214.

21. Cranston HA, Boylan CW, Carroll GL, Sutera SP, Gluzman IY, Krogstad DJ. Plasmodium falciparum maturation abolishes physiologic red cell deformability. Science. 1984;223(4634):400-403.

22. Suresh S, Spatz J, Mills JP, Micoulet A, Dao M, Lim CT, et al. Connections between single-cell biomechanics and human disease states: gastrointestinal cancer and malaria. Acta Biomaterialia. 2005;1(1):15-30. doi:10.1016/j.actbio.2004.09.001.

23. Park Y, Diez-Silva M, Popescu G, Lykotrafitis G, Choi W, Feld MS, et al. Refractive index maps and membrane dynamics of human red blood cells parasitized by Plasmodium falciparum. Proceedings of the National Academy of Sciences. 2008;105(37):13730-13735. doi:10.1073/pnas.0806100105.

24. Gruenberg J, Allred DR, Sherman IW. Scanning electron microscope-analysis of the protrusions (knobs) present on the surface of Plasmodium falciparum-infected erythrocytes. The Journal of cell biology. 1983;97(3):795-802.

25. Quadt KA, Barfod L, Andersen D, Bruun J, Gyan B, Hassenkam T, et al. The density of knobs on Plasmodium falciparum-infected erythrocytes depends on developmental age and varies among isolates. PLoS One. 2012;7(9):e45658.

26. Crabb BS, Cooke BM, Reeder JC, Waller RF, Caruana SR, Davern KM, et al. Targeted gene disruption shows that knobs enable malaria-infected red cells to cytoadhere under physiological shear stress. Cell. 1997;89(2):287-296. doi:10.1016/s0092-8674(00)80207-x. 
27. Oh SS, Voigt S, Fisher D, Scott JY, LeRoy PJ, Derick LH, et al. Plasmodium falciparum erythrocyte membrane protein 1 is anchored to the actin-spectrin junction and knob-associated histidine-rich protein in the erythrocyte skeleton. Molecular and biochemical parasitology. 2000;108(2):237-247.

28. Cutts EE, Laasch N, Reiter DM, Trenker R, Slater LM, Stansfeld PJ, et al. Structural analysis of P. falciparum KAHRP and PfEMP1 complexes with host erythrocyte spectrin suggests a model for cytoadherent knob protrusions. PLOS Pathogens. 2017;13(8):e1006552. doi:10.1371/journal.ppat.1006552.

29. Watermeyer JM, Hale VL, Hackett F, Clare DK, Cutts EE, Vakonakis I, et al. A spiral scaffold underlies cytoadherent knobs in Plasmodium falciparum-infected erythrocytes. Blood. 2016;127(3):343-351.

30. Looker O, Blanch AJ, Liu B, Nunez-Iglesias J, McMillan PJ, Tilley L, et al. The knob protein KAHRP assembles into a ring-shaped structure that underpins virulence complex assembly. PLoS pathogens. 2019;15(5):e1007761.

31. Sanchez CP, Karathanasis C, Sanchez R, Cyrklaff M, Jaeger J, Buchholz B, et al. Single-molecule imaging and quantification of the immune-variant adhesin VAR2CSA on knobs of Plasmodium falciparum -infected erythrocytes. Communications Biology. 2019;2(1):1-9. doi:10.1038/s42003-019-0429-z.

32. Shi H, Liu Z, Li A, Yin J, Chong AG, Tan KS, et al. Life cycle-dependent cytoskeletal modifications in Plasmodium falciparum infected erythrocytes. PLoS One. 2013;8(4):e61170.

33. Zhang Y, Huang C, Kim S, Golkaram M, Dixon MW, Tilley L, et al. Multiple stiffening effects of nanoscale knobs on human red blood cells infected with Plasmodium falciparum malaria parasite. Proceedings of the National Academy of Sciences. 2015;112(19):6068-6073.

34. Sanchez CP, Patra P, Chang SYS, Karathanasis C, Hanebutte L, Kilian N, et al. KAHRP dynamically relocalizes to remodeled actin junctions and associates with knob spirals in P. falciparum-infected erythrocytes. Molecular Microbiology. 2021;n/a(n/a). doi:https://doi.org/10.1111/mmi.14811.

35. Li J, Lykotrafitis G, Dao M, Suresh S. Cytoskeletal dynamics of human erythrocyte. Proceedings of the National Academy of Sciences. 2007;104(12):4937-4942. doi:10.1073/pnas.0700257104.

36. Chang HY, Li X, Li H, Karniadakis GE. MD/DPD multiscale framework for predicting morphology and stresses of red blood cells in health and disease. PLoS Computational Biology. 2016;12(10):e1005173.

37. Hoffmann M, Fröhner C, Noé F. ReaDDy 2: Fast and flexible software framework for interacting-particle reaction dynamics. PLOS Computational Biology. 2019;15(2):e1006830. doi:10.1371/journal.pcbi.1006830.

38. Tomishige M, Sako Y, Kusumi A. Regulation mechanism of the lateral diffusion of band 3 in erythrocyte membranes by the membrane skeleton. The Journal of cell biology. 1998;142(4):989-1000.

39. Lanni F, Ware BR. Detection and characterization of actin monomers, oligomers, and filaments in solution by measurement of fluorescence photobleaching recovery. Biophysical Journal. 1984;46(1):97-110. 
40. Kilejian A, Rashid MA, Aikawa M, Aji T, Yang YF. Selective association of a fragment of the knob protein with spectrin, actin and the red cell membrane. Molecular and Biochemical Parasitology. 1991;44(2):175-181. doi:10.1016/0166-6851(91)90003-O.

41. Pei X, An X, Guo X, Tarnawski M, Coppel R, Mohandas N. Structural and functional studies of interaction between Plasmodium falciparum knob-associated histidine-rich protein (KAHRP) and erythrocyte spectrin. Journal of Biological Chemistry. 2005;.

42. Rug M, Prescott SW, Fernandez KM, Cooke BM, Cowman AF. The role of KAHRP domains in knob formation and cytoadherence of $\mathrm{P}$ falciparum-infected human erythrocytes. Blood. 2006;108(1):370-378.

43. Weng H, Guo X, Papoin J, Wang J, Coppel R, Mohandas N, et al. Interaction of Plasmodium falciparum knob-associated histidine-rich protein (KAHRP) with erythrocyte ankyrin $\mathrm{R}$ is required for its attachment to the erythrocyte membrane. Biochimica et biophysica acta. 2014;1838(1 0 0):185-192. doi:10.1016/j.bbamem.2013.09.014.

44. Magowan C, Nunomura W, Waller KL, Yeung J, Liang J, Van Dort H, et al. Plasmodium falciparum histidine-rich protein 1 associates with the band 3 binding domain of ankyrin in the infected red cell membrane. Biochimica et Biophysica Acta (BBA)-Molecular Basis of Disease. 2000;1502(3):461-470.

45. Hall R, Dixon T, Dickson A. On Calculating Free Energy Differences Using Ensembles of Transition Paths. Frontiers in Molecular Biosciences. 2020;7. doi:10.3389/fmolb.2020.00106.

46. Guo K, Shillcock J, Lipowsky R. Self-assembly of actin monomers into long filaments: Brownian dynamics simulations. The Journal of Chemical Physics. 2009;131(1):015102. doi:10.1063/1.3159003.

47. Humphrey W, Dalke A, Schulten K. VMD - Visual Molecular Dynamics. Journal of Molecular Graphics. 1996;14:33-38.

48. Schnitzbauer J, Wang Y, Zhao S, Bakalar M, Nuwal T, Chen B, et al. Correlation analysis framework for localization-based superresolution microscopy. Proceedings of the National Academy of Sciences. 2018;115(13):3219-3224.

49. Sengupta P, Jovanovic-Talisman T, Skoko D, Renz M, Veatch SL, Lippincott-Schwartz J. Probing protein heterogeneity in the plasma membrane using PALM and pair correlation analysis. Nature methods. 2011;8(11):969-975.

50. Li H, Lykotrafitis G. Erythrocyte membrane model with explicit description of the lipid bilayer and the spectrin network. Biophysical journal. 2014;107(3):642-653.

51. Min K, Silberstein M, Aluru NR. Crosslinking PMMA: Molecular dynamics investigation of the shear response. Journal of Polymer Science Part B: Polymer Physics. 2014;52(6):444-449. doi:https://doi.org/10.1002/polb.23437.

52. Feng Z, Waugh RE, Peng Z. Constitutive Model of Erythrocyte Membranes with Distributions of Spectrin Orientations and Lengths. Biophysical Journal. 2020;119(11):2190-2204. doi:10.1016/j.bpj.2020.10.025. 
53. Zhu Q, Vera C, Asaro RJ, Sche P, Sung LA. A hybrid model for erythrocyte membrane: a single unit of protein network coupled with lipid bilayer.

Biophysical journal. 2007;93(2):386-400.

54. Lee JCM, Discher DE. Deformation-Enhanced Fluctuations in the Red Cell Skeleton with Theoretical Relations to Elasticity, Connectivity, and Spectrin Unfolding. Biophysical Journal. 2001;81(6):3178-3192. doi:10.1016/S0006-3495(01)75954-1.

55. Peng Z, Asaro RJ, Zhu Q. Multiscale simulation of erythrocyte membranes. Physical Review E. 2010;81(3):031904.

56. Pease BN, Huttlin EL, Jedrychowski MP, Talevich E, Harmon J, Dillman T, et al. Global analysis of protein expression and phosphorylation of three stages of Plasmodium falciparum intraerythrocytic development. Journal of Proteome Research. 2013;12(9):4028-4045. doi:10.1021/pr400394g.

57. Cobbold SA, Santos JM, Ochoa A, Perlman DH, Llinás M. Proteome-wide analysis reveals widespread lysine acetylation of major protein complexes in the malaria parasite. Scientific Reports. 2016;6(1):19722. doi:10.1038/srep19722.

58. Pollard TD. Rate constants for the reactions of ATP- and ADP-actin with the ends of actin filaments. The Journal of Cell Biology. 1986;103(6):2747-2754. doi:10.1083/jcb.103.6.2747. 